ORNL/TM-2015/238

\title{
Mining Archived HYSPEC User Data to Analyze the Prompt Pulse at the SNS
}

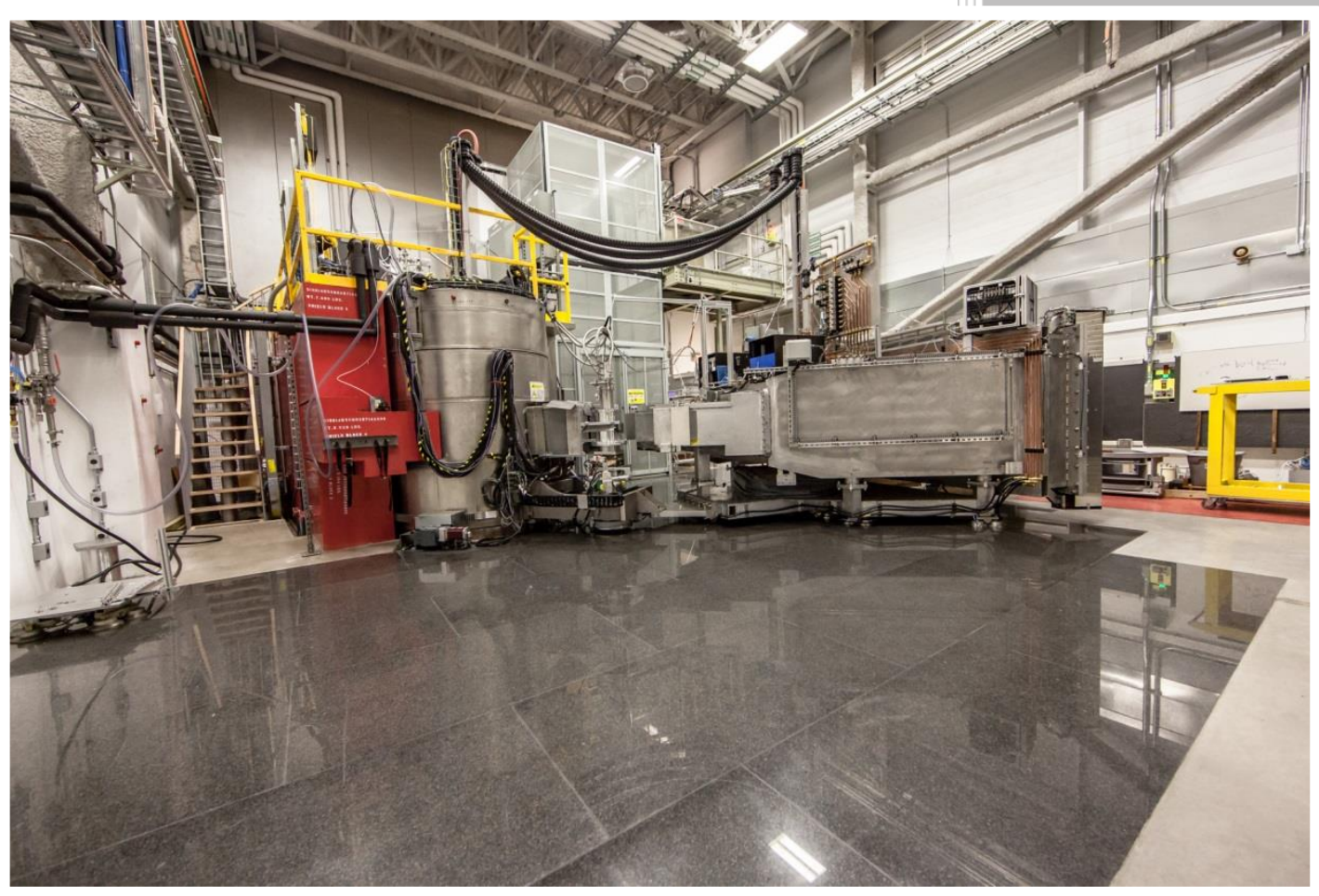

Michael B. R. Smith Erik B. Iverson Franz X. Gallmeier Barry L. Winn

$10 / 1 / 2015$

Approved for public release. Distribution is unlimited. 


\section{DOCUMENT AVAILABILITY}

Reports produced after January 1, 1996, are generally available free via US Department of Energy (DOE) SciTech Connect.

Website http://www.osti.gov/scitech/

Reports produced before January 1, 1996, may be purchased by members of the public from the following source:

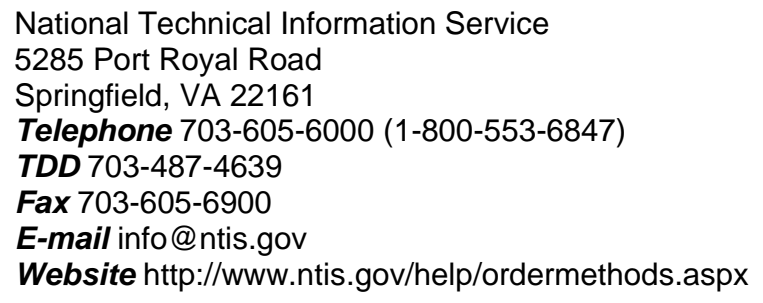

Reports are available to DOE employees, DOE contractors, Energy Technology Data Exchange representatives, and International Nuclear Information System representatives from the following source:

Office of Scientific and Technical Information

PO Box 62

Oak Ridge, TN 37831

Telephone 865-576-8401

Fax 865-576-5728

E-mail reports@osti.gov

Website http://www.osti.gov/contact.html

This report was prepared as an account of work sponsored by an agency of the United States Government. Neither the United States Government nor any agency thereof, nor any of their employees, makes any warranty, express or implied, or assumes any legal liability or responsibility for the accuracy, completeness, or usefulness of any information, apparatus, product, or process disclosed, or represents that its use would not infringe privately owned rights. Reference herein to any specific commercial product, process, or service by trade name, trademark, manufacturer, or otherwise, does not necessarily constitute or imply its endorsement, recommendation, or favoring by the United States Government or any agency thereof. The views and opinions of authors expressed herein do not necessarily state or reflect those of the United States Government or any agency thereof. 
Instrument and Source Division

\title{
MINING ARCHIVED HYSPEC USER DATA TO ANALYZE THE PROMPT PULSE AT THE SPALLATION NEURTON SOURCE
}

Michael B. R. Smith

Erik B. Iverson

Franz X. Gallmeier

Barry L. Winn

Date Published: October 2015

\author{
Prepared by \\ OAK RIDGE NATIONAL LABORATORY \\ Oak Ridge, Tennessee 37831-6283 \\ managed by \\ UT-BATTELLE, LLC \\ for the \\ US DEPARTMENT OF ENERGY \\ under contract DE-AC05-00OR22725
}





\section{CONTENTS}

LIST OF FIGURES
LIST OF TABLES.
ACRONYMS
1.
BACKGROUND AND INTRODUCTION





\section{LIST OF FIGURES}

Figure

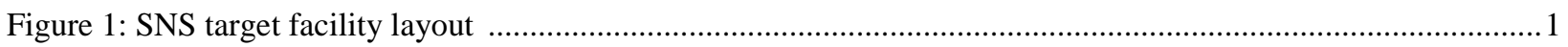

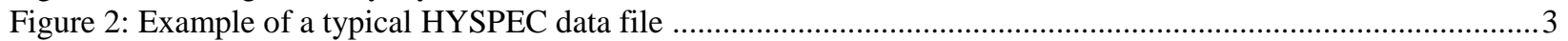

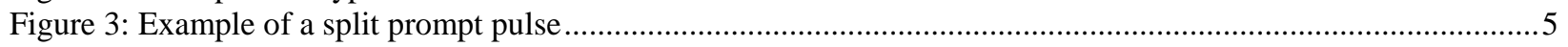

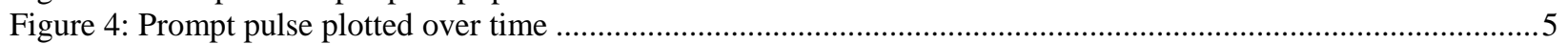

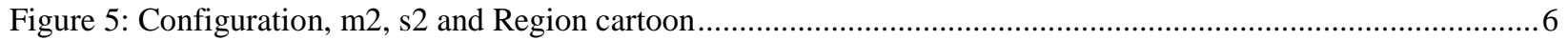

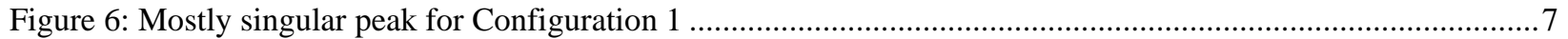

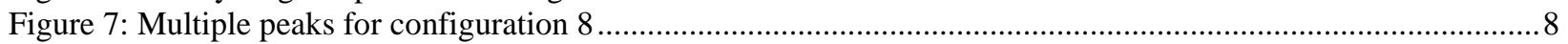

Figure 8: Drop in HYSPEC background after beam outage and shielding installation .............................................. 11

Figure 9: Beamline 13 shutter correlation with HYSPEC prompt pulse magnitude .............................................. 12

Figure 10: HYSPEC configurations and beamline 13 shutter effects .................................................................. 13

Figure 11: Large discrepancy between prompt pulse magnitudes shifting as a function of configuration while

BL13 shutter is open and all other North side shutters are open. ................................................................ 14

Figure 12: Prompt pulse magnitudes not shifting as a function of configuration while BL13 shutter is closed

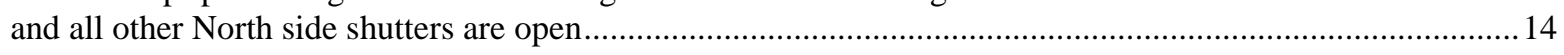

Figure 13: Arithmetic difference of pulse shapes for different instrument configurations ........................................ 16

Figure 14: Contributions to HYSPEC prompt pulse magnitude as a percentage of average pulse when all

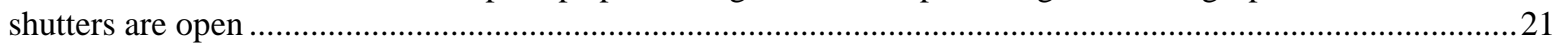

Figure 15: Hypothetical prompt pulse with all identified contributors eliminated compared to a typical prompt pulse with all shutters in their maximum adverse configuration .................................................................22

Figure 16: Beamline shutter contributing to prompt pulse shape change ...............................................................23

Figure 17: Example of reasonable detector vessel data .................................................................................... B-1

Figure 18: Example of unreasonable detector vessel data .................................................................................. B-1

Figure 19: Maximum TOF window expected from elastic data compared to total TOF window for data

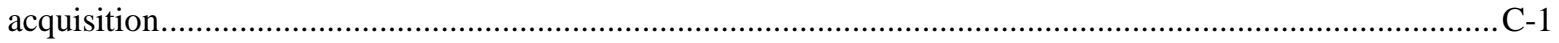

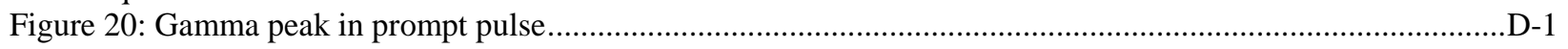

Figure 21: Reconstruction of split prompt pulse ............................................................................................. E-1

Figure 22: Vector names with "head" and "tail" coordinate locations for orientation ............................................ F-2

Figure 23: HYSPEC in configuration 1. Comparing background levels when all shutters on the north side of the target facility were open to when certain beamlines were the only ones closed .......................................... J-1

Figure 24: HYSPEC in configuration 3. Comparing background levels when all shutters on the north side of the target facility were open to when certain beamlines were the only ones closed .......................................... J-2

Figure 25: HYSPEC in configuration 3. Comparing background levels when all shutters on the north side of the target facility were open to when certain beamlines were the only ones closed....................................... J-2

Figure 26: HYSPEC in configuration 5. Comparing background levels when all shutters on the north side of the target facility were open to when certain beamlines were the only ones closed. .........................................J-3

Figure 27: HYSPEC in configuration 5. Comparing background levels when all shutters on the north side of the target facility were open to when certain beamlines were the only ones closed. ........................................ J-3

Figure 28: HYSPEC in configuration 6. Comparing background levels when all shutters on the north side of the target facility were open to when certain beamlines were the only ones closed.......................................... J-4

Figure 29: HYSPEC in configuration 6. Comparing background levels when all shutters on the north side of the target facility were open to when certain beamlines were the only ones closed. ........................................ J-4

Figure 30: HYSPEC in configuration 8. Comparing background levels when all shutters on the north side of the target facility were open to when certain beamlines were the only ones closed. ......................................... J-5 



\section{LIST OF TABLES}

\section{Table}

Table 1: Script filter names, descriptions and references .4

Table 2: Explanation of statistical symbols and terminology

Table 3: Pearson-Chi Square results for HYSPEC instrument configuration comparisons ..................................15

Table 4: HYSPEC background signature while specific shutter is the only shutter closed on the north side of the target facility

Table 5: Difference in prompt pulse magnitude while the specific shutter is the only one closed and when all the

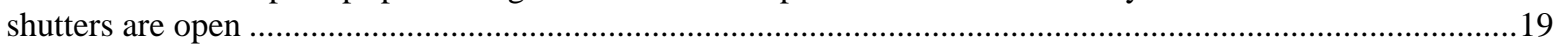

Table 6: Average portion of the prompt pulse magnitude across all HYSPEC configurations for all north side beamlines .21

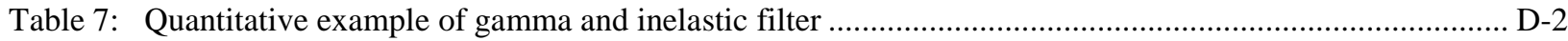

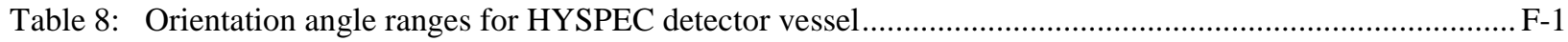

Table 9: Calculations for prompt pulse magnitude when all north side shutters are open .................................. I-2

Table 10: Calculations for prompt pulse magnitude where BL-11 primary shutter is the only one closed ............. I-3

Table 11: Calculations for prompt pulse magnitude where BL-11a secondary shutter is the only one closed ......... I-3

Table 12: Calculations for prompt pulse magnitude where BL-11b secondary shutter is the only one closed......... I-3

Table 13: Calculations for prompt pulse magnitude where BL-12 primary shutter is the only one closed .............. I-4

Table 14: Calculations for prompt pulse magnitude where BL-13 primary shutter is the only one closed .............. I-4

Table 15: Calculations for prompt pulse magnitude where BL-13 secondary shutter is the only one closed........... I-4

Table 16: Calculations for prompt pulse magnitude where BL-15 primary shutter is the only one closed ............. I-5

Table 17: Calculations for prompt pulse magnitude where BL-15 secondary shutter is the only one closed........... I-5

Table 18: Calculations for prompt pulse magnitude where BL-16 primary shutter is the only one closed ............. I-5

Table 19: Calculations for prompt pulse magnitude where BL-16 secondary shutter is the only one closed.......... I-6

Table 20: Calculations for prompt pulse magnitude where BL-17 primary shutter is the only one closed ............. I-6

Table 21: Calculations for prompt pulse magnitude where BL-18 primary shutter is the only one closed .............. I-6 



\section{ACRONYMS}

$\begin{array}{ll}\text { SNS } & \text { Spallation Neutron Source } \\ \text { HYSPEC } & \text { Hybrid Spectrometer } \\ \text { ORNL } & \text { Oak Ridge National Laboratories } \\ \text { TOF } & \text { time of flight } \\ \text { CSS } & \text { Control Systems Studio } \\ \text { PV } & \text { Process Variable } \\ \text { CSV } & \text { comma seperated value }\end{array}$





\section{BACKGROUND AND INTRODUCTION}

The Hybrid-Spectrometer (HYSPEC) [1] is one of 17 instruments currently operated at the Spallation Neutron Source (SNS) at Oak Ridge National Laboratories (ORNL). The secondary spectrometer of this instrument is located inside an out-building off the north side of the SNS instrument hall [Figure 1]. HYSPEC has experienced a larger background feature than similar inelastic instruments since its commissioning in 2011. This background feature is caused by a phenomenon known as the "prompt pulse" which is an essential part of neutron production in a pulsed spallation source but comes with unfortunate side effects.

\subsection{GENERAL TARGET FACILITY INFORMATION}

The SNS is a pulsed neutron source that generates its neutrons by subjecting a liquid mercury target to a $60 \mathrm{~Hz}$ proton beam. The protons collide with the target material, creating spallation events, and liberate a broad spectrum of neutron energies for scientific use in the various beamlines.

Despite the fact that the target, beamline flight-paths, shutters and beam stops are all enclosed in large shielding structures, small portions of the higher energy neutrons can and do escape into the target building. The neutrons that escape can then slow down by interaction with numerous materials or spread in time by time-of-flight dispersion. These neutrons create a $60 \mathrm{~Hz}$ pulsed background signature (the prompt pulse) that arrives at consistent $16.667 \mathrm{~ms}$ intervals $\left(1 / 60^{\text {th }}\right.$ of a second $)$ and can be detected by sensitive instruments like HYSPEC.

\subsection{SPECIFIC INFORMATION}

HYSPEC

The discovery of the large magnitude and duration of the prompt pulse for HYSPEC was unsettling and past experiments have been conducted by beamline staff to identify potential opportunities for mitigation or elimination of this phenomenon.

Although specific contributors to prompt pulse fluctuations were identified in isolated tests in the past, a comprehensive analysis over the life of the instrument has now been undertaken to provide a more accurate characterization of prompt pulse variations as seen by HYSPEC.

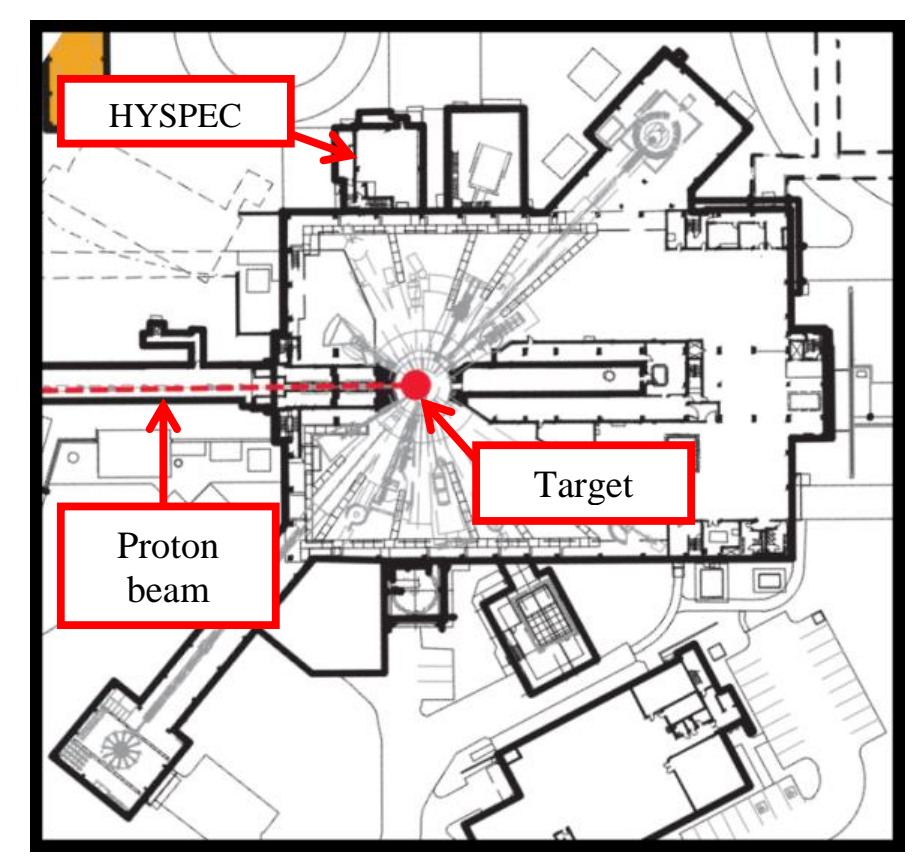

Figure 1: SNS target facility layout. 



\section{METHODS}

\subsection{ARCHIVED HYSPEC DATA}

To illustrate the effect of the prompt pulse, a useful format for viewing data from an archived experiment at HYSPEC is a histogrammed series of normalized counts (cnts/ $\mu \mathrm{Ahr}$ ) over a $16.667 \mathrm{~ms}$ window of time [Figure 2]. This neutron data is accompanied by a run log containing other relevant instrument information regarding aspects of the cumulative proton beam charge, duration of run, angle(s) of detector vessel, dates and much more. All of this information is contained within a single Nexus (.nxs) file which is a specific file format designed for Manitdplot [2] and other neutron scattering software.

Since the expected time-of-flight (TOF) for the elastic peak can be predicted to high accuracy, the window for the data collection is centered on this specific time and the user's inelastic data will typically precede (kinetic energy gain) or follow (kinetic energy loss) this peak within the pre-allocated TOF window.

The limiting of the time window to $16.667 \mathrm{~ms}$ is chosen so that neutrons from a single proton pulse are the only ones observed in the data. The possibility of similar detection times of neutrons with different speeds and from different pulses can destroy the required one-to-one relationship between timeof-arrival and time-of-flight. This window width is controlled by bandwidth choppers that allow neutrons to pass only at a time window determined by the particular phase chosen for the choppers. In addition, using a direct geometry spectrometer strategy, a narrow range of incident energies can be selected and the energy transferred can be measured via varying final detection times.

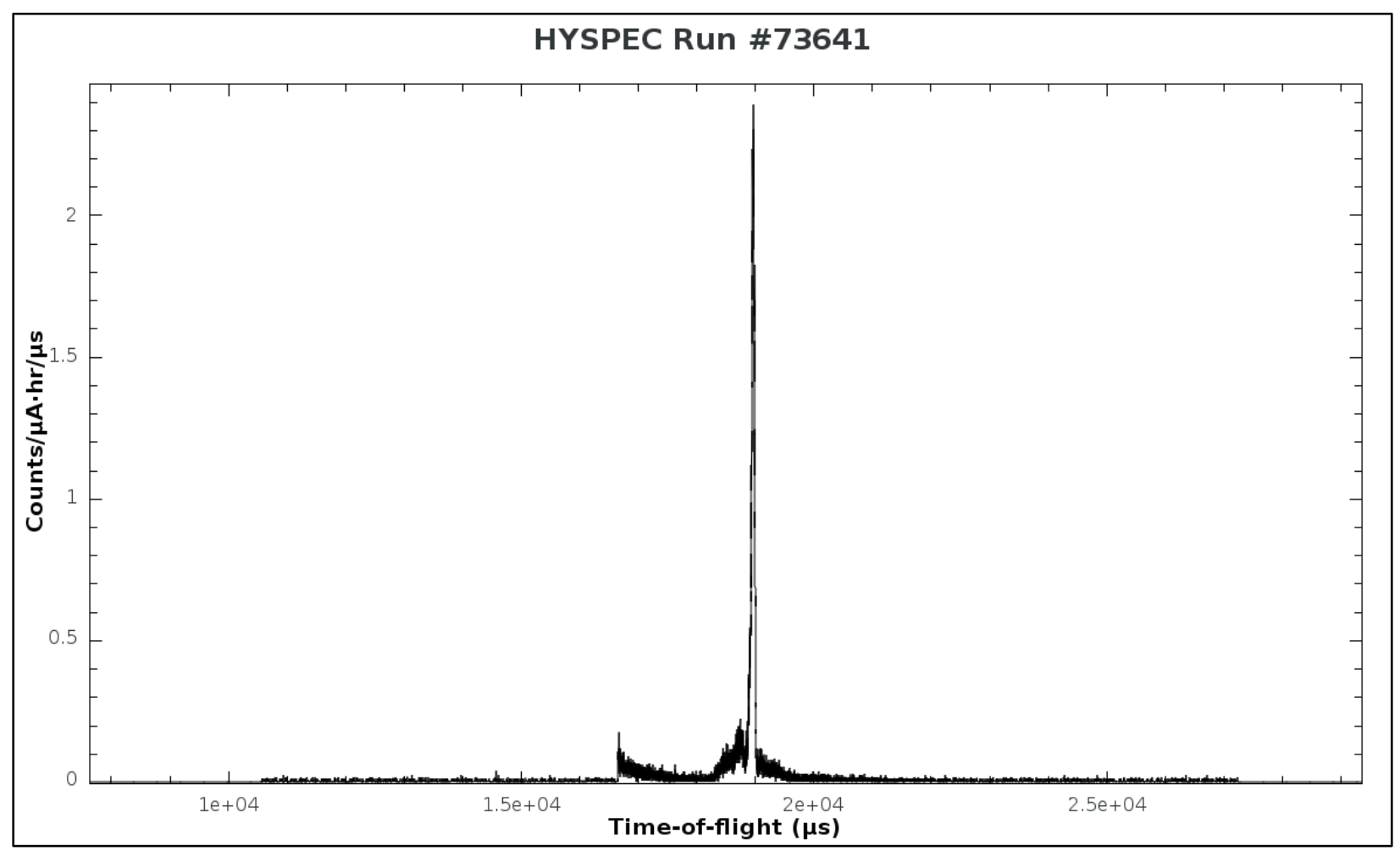

Figure 2: Example of a typical HYSPEC data file. 


\subsection{DEVELOPING THE ANALYSIS}

We developed a data mining approach to get a complete, objective look at long and short term trends in the HYSPEC prompt pulse. This approach accessed some 60,000 archived user data files using Mantidplot, which is a software package designed to effectively and efficiently access SNS neutron scattering instrument data. Within this software, Python [3] scripts were created that accessed the files of archived user data. Using these custom Python algorithms and preexisting algorithms and toolboxes within Mantidplot, the data was accessed and manipulated to fit the purposes of this analysis.

In approaching a large-scale methodology for mining these data sets, or "runs", various parameters regarding what was acceptable and unacceptable for this analysis needed to be established prior to including or excluding a run in the investigation. This led to a filtering process that excluded runs that reflected various undesirable traits from the examination. A table of filtering parameters is listed below with details regarding the specifics of the scripting and quantitative values explained in the respective appendices [Table 1].

Table 1: Script filter names, descriptions and references

\begin{tabular}{lll}
\hline Filter Name & Brief Description & Detailed Description \\
\hline Duration and/or Charge Filter & $\begin{array}{l}\text { Excludes runs with insufficient run } \\
\text { time and/or total charge to provide } \\
\text { statistics worthy of analysis }\end{array}$ & \\
Detector Location Filter & $\begin{array}{l}\text { Excludes runs that have erroneous } \\
\text { location data and/or multiple } \\
\text { positions }\end{array}$ & Appendix B \\
Elastic/Inelastic Peak Filter & $\begin{array}{l}\text { Excludes runs where the prompt Appendix C } \\
\text { pulse could be obscured by the } \\
\text { user's elastic/inelastic data }\end{array}$ & \\
Gamma Peak Filter & $\begin{array}{l}\text { Excludes runs where the tail of the } \\
\text { prompt pulse indicates that there is a } \\
\text { gamma peak present in the prompt } \\
\text { pulse }\end{array}$ & \\
\hline
\end{tabular}

After the filtering process was applied to all the available data sets, the analysis was left with approximately 24,000 runs from the original 60,000 runs (nearly 60\% excluded).

We chose a metric for assessing the impact of the prompt pulse as the integral of the normalized count-rate over a $3 \mathrm{~ms}$ window beginning at the onset of the prompt pulse. This $3 \mathrm{~ms}$ window regularly straddles the frame boundary - that is, the prompt pulse is split between the beginning and end of the time-of-flight frame, requiring that we identify split pulses and correctly integrate both portions [Figure 3]. Specifics of this algorithm are explained in greater detail in Appendix E. 


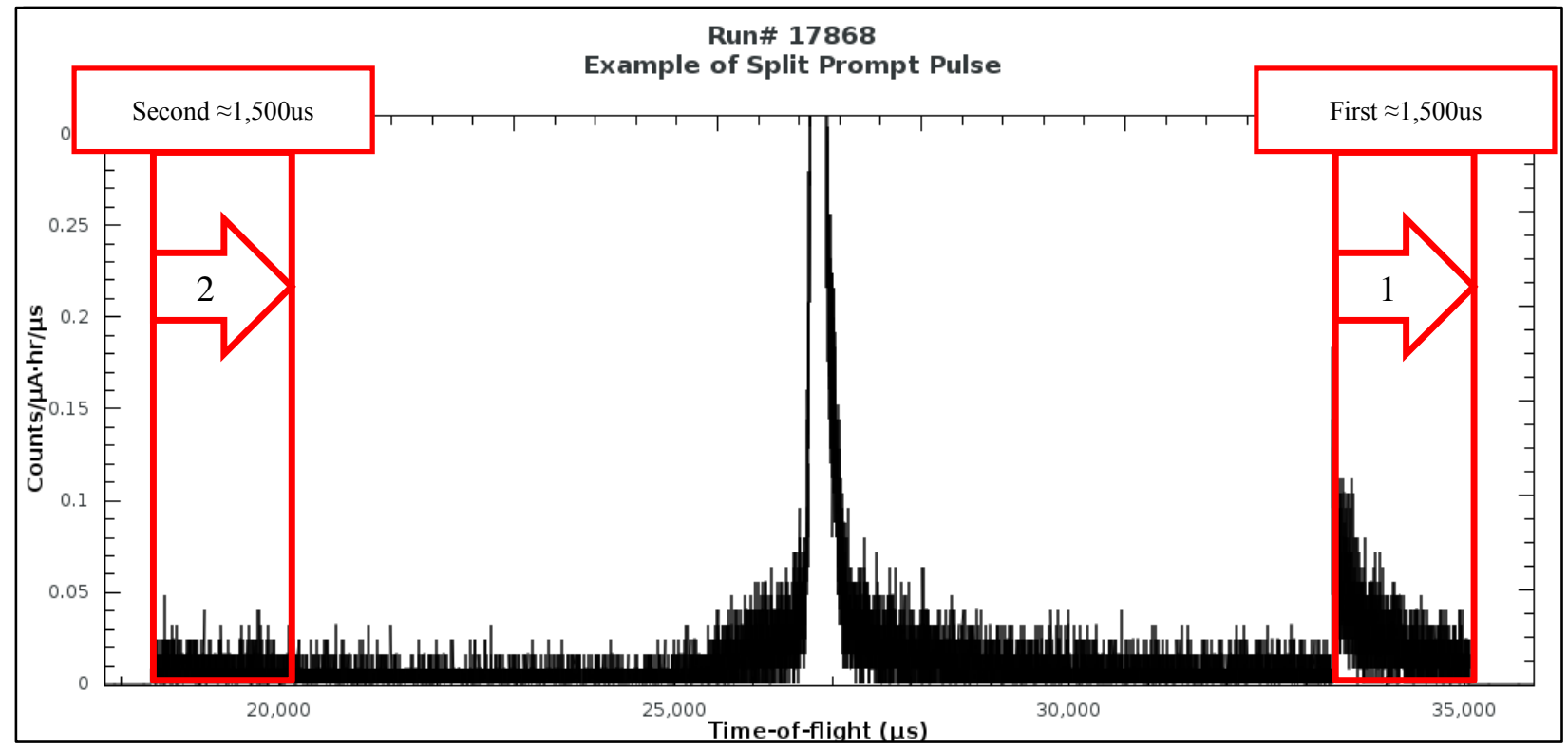

Figure 3: Example of a split prompt pulse.

The magnitude of the prompt pulse (as integrated over $3 \mathrm{~ms}$ ) can then be plotted as a function of run number, date or time depending on the desired effect [Figure 4]. Once this was performed, a visual representation of the relative magnitudes of the prompt pulses could be observed over time. This quickly revealed that not only were there distinct short term fluctuations but also long term trends to be investigated.

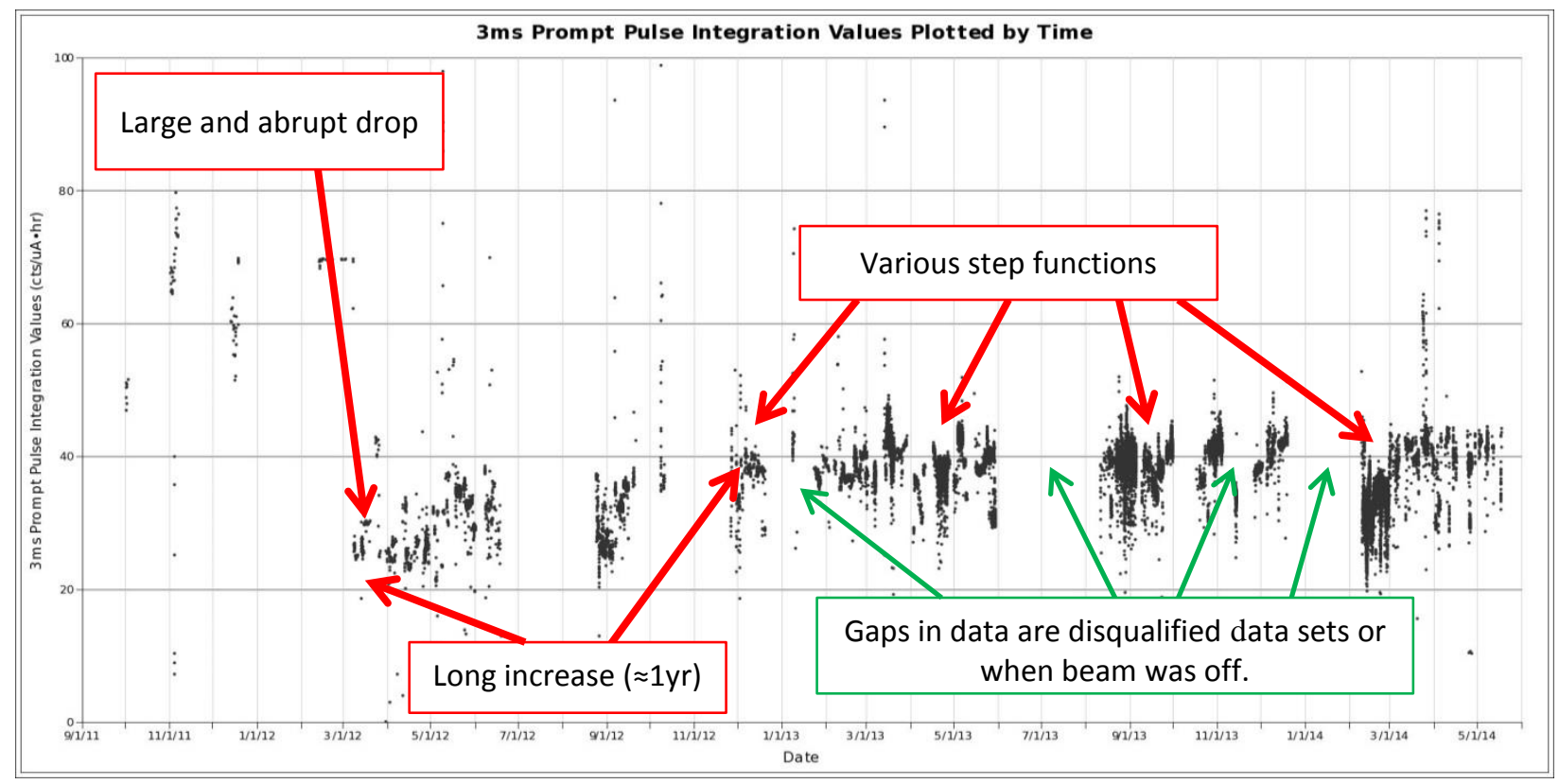

Figure 4: Prompt pulse plotted over time. 


\subsection{INSTRUMENT CONFIGURATION}

The observation of consistent fluctuations in magnitude required the analysis to identify and isolate variables that could cause these variations in the prompt pulse for HYSPEC. The first known variable needing to be addressed was that the detector vessel for HYSPEC can move about the room. HYSPEC has two pivoting axes. One axis is about the monochromating crystal array and the other is about the sample, known in the log data as "m2" and "s2" respectively. With the known length of the flight path between $\mathrm{m} 2$ and s2 and the known length between s2 and the detector array, a trigonometric algorithm was created to categorize the various angles of the detector array along with the physical location in the room at the time of each run.

This

distinction needed to be made as the fact that the instrument has multiple axes means that simply identifying where the detector was in the room wasn't sufficient, how the instrument was in

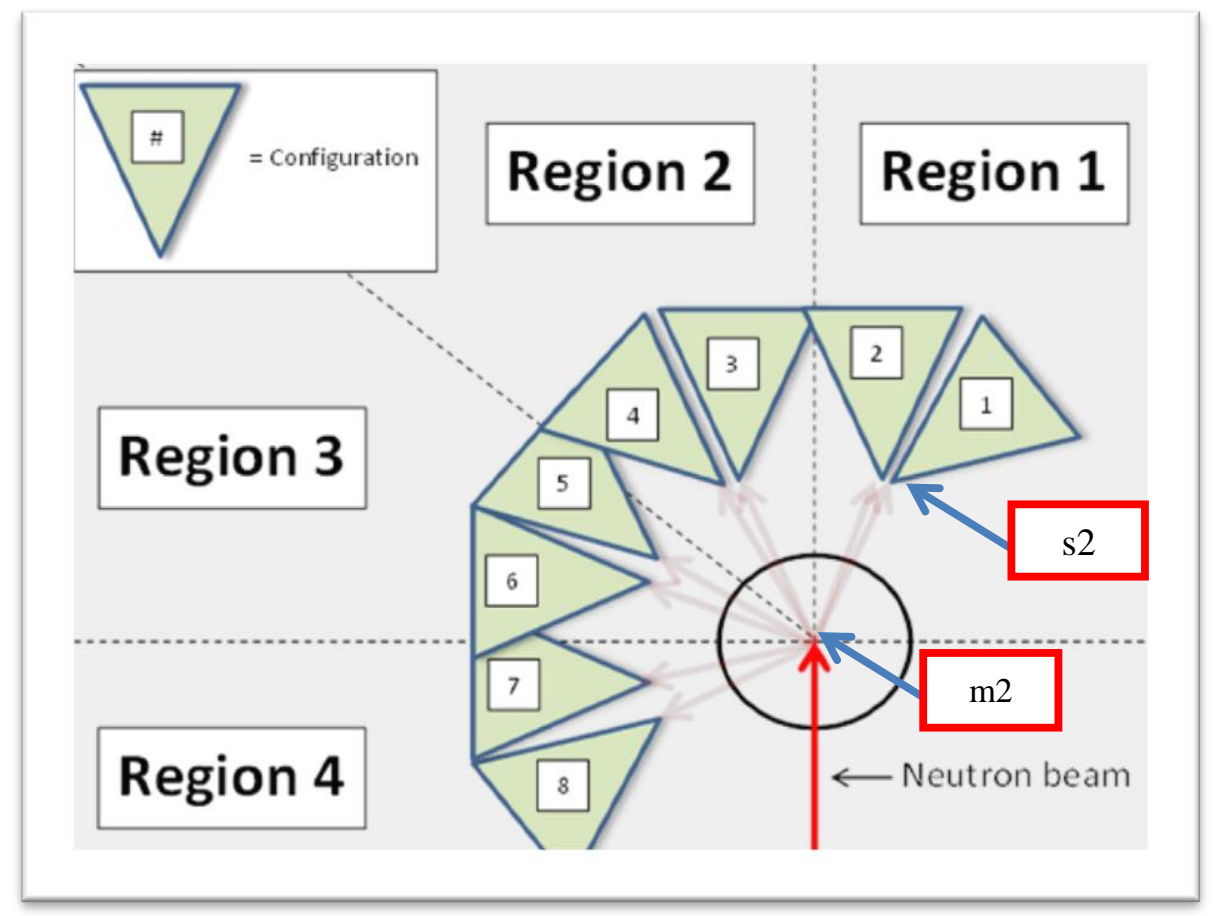
the room also needed to be addressed.

For the sake of this analysis, specific terminology was assigned to describe these two features. "Region" was used to describe where the detector vessel was while "orientation" was used to describe how it was situated. Understanding all the physical restraints on the machine and all the possible classifications of region and orientation a new term was assigned that encompassed both of these critical factors. "Configuration" was decided to explain both where and how the vessel was located in the room and a total of 8 configurations were found to be achievable described by the parameters of this analysis. To define these parameters, the room was arbitrarily broken into four regions centered on $\mathrm{m} 2$ [Figure 5]. The center of the detector array was selected as the point to determine the region, and the relative angle of the detector array in relation to a fixed point was used to determine the orientation of the vessel. Details regarding the confirmation of this algorithm's accuracy and the qualification process can be found in Appendix F. 


\subsection{FREQUENCY DISTRIBUTIONS OF DATA}

Once the classification of the configuration was proven to be consistent and accurate the data could be further sub-grouped not only by the magnitude of the prompt pulse but also by the configuration at the time of data collection. This allowed us to view variations in the prompt pulse while holding the configuration of the instrument constant. Grouping the data like this allowed further scrutiny by frequency distribution of the prompt pulse magnitudes for every individual configuration.

These frequency distributions were generated to see if there were any obvious correlations between the magnitude of the prompt pulse and the configuration of the vessel. When plotted, these distributions showed multiple points of interest. For certain configurations there were singular peaks implying limited external influence on the prompt pulse [Figure 6] while other configurations showed multiple peaks (with configuration held constant) implying that something external was causing variations in the magnitude [Figure 7]. Further detail regarding the scripting and controlling algorithms for this process can be found in Appendix $G$ and various illustrative frequency distributions can be found in Appendix J.

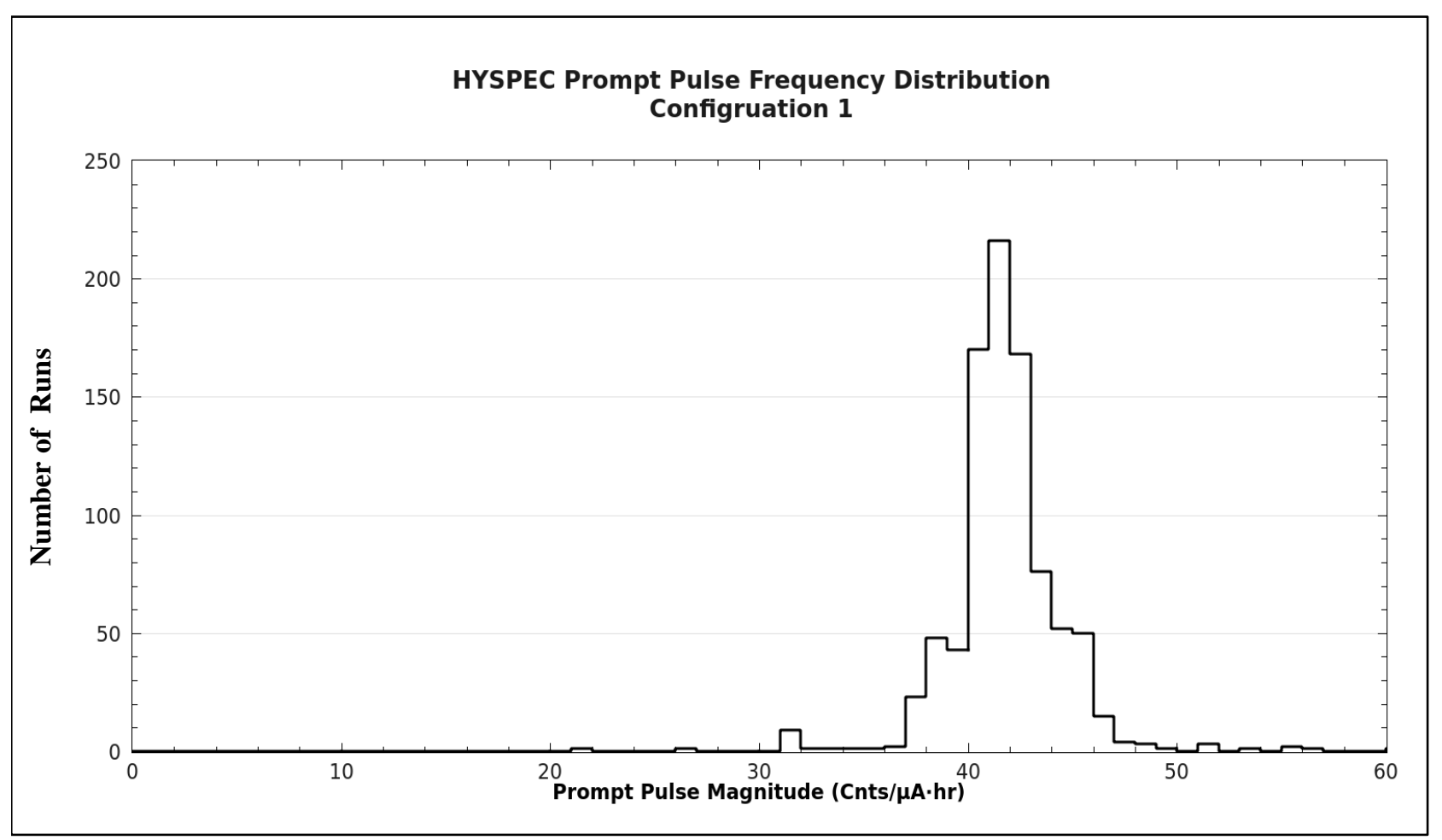

Figure 6: Configuration 1 shows unimodal peak. 


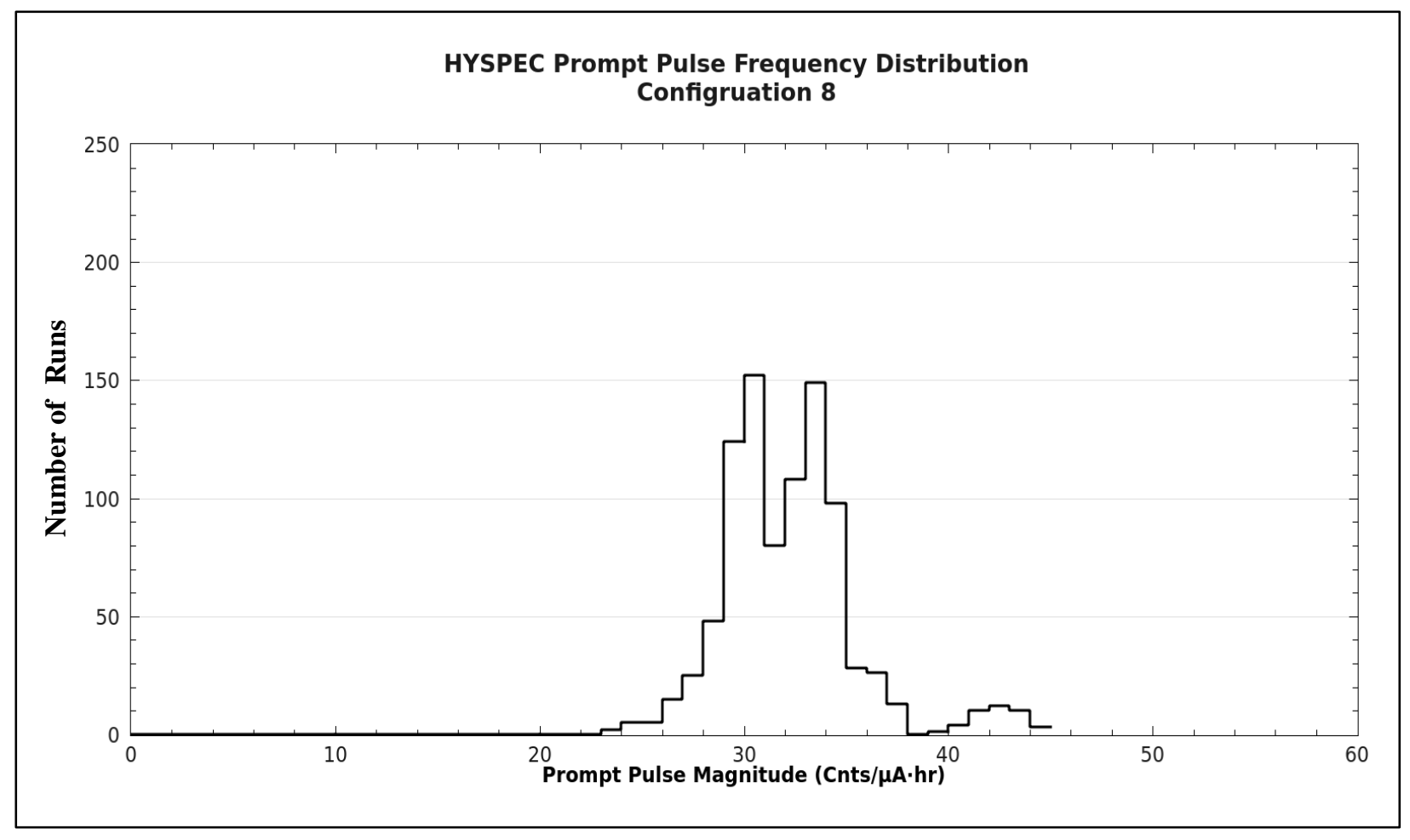

Figure 7: Configuration 8 shows multimodal distribution.

With HYSPEC configurations accounted for, the external influences could be further investigated. HYSPEC beamline staff had investigated fluctuations in the prompt pulse caused by beamline shutter positions of neighboring instruments in the past. We repeated this investigation systematically over the life of the instrument to confirm and/or refute these affects. Shutter position data was accessed through the Control Systems Studio (CSS) [4] used at the SNS for accessing and managing any available Process Variables (PV) either in real-time or from archived data. Specific shutter data was loaded for desired dates from 2011 to the current day (being the day of analysis) and exported back to Mantidplot for further analysis.

These data could then be applied to this analysis by further classifying the prompt pulse magnitude by the configuration of HYSPEC along with the position of neighboring shutters. Once this task was performed the frequency distributions of each configuration could be recalculated and analyzed with the shutter variables held constant. In trying to isolate the influence of shutter positions, the initial assumption was to observe all the analyzed runs while all shutters were closed except the one in question. It became quickly evident that this was a rare, if not unheard of, arrangement for the facility as when the beam is on, all instruments are hoping to be operational and thus having their shutters open. Because of this fact, the contribution of each shutter was deduced by comparing the data collected while a specific shutter was the only shutter open on the north side of the instrument hall. This value was then compared to the background magnitude when all the north side shutters were open. The difference of these two values was deemed the individual contribution of each shutter and served as the mean or 'canonical' pulse magnitude for all particular combinations of instrument configuration and target facility shutter configurations.

Details regarding the importing of data, handling of data and controlling algorithms for this process can be found in Appendix $\mathrm{H}$. 


\subsection{PULSE SHAPE}

Since the nature of the data being analyzed was time-of-flight (TOF) data, the pulse shape (not just the magnitude) was of interest to help further characterize representative pulses for each instrument and shutter configuration combination.

To identify changes in pulse shape without taking into account differences in pulse magnitudes, every prompt pulse evaluated was normalized to unit area. Then, the normalized pulses were classified by specific configuration criteria (instrument configuration and shutter configuration). To confirm the homogeneity of each combination of configuration criteria a test group of runs were randomly sampled for each configuration criteria and compared to other randomly selected runs of the same configuration criteria via a Pearson-Chi Square test. These tests showed the samples to be statistically consistent in shape within each of these tested shutter and instrument configurations. Each pulse shape representing a particular configuration combination was then averaged over the sample set to develop a "mean" or "canonical" pulse shape per unit area. These various canonical pulse shapes could then be compared to each other statistically and arithmetically to determine if the shape fluctuated as a function of configuration and shutter position and if so, where in time these differences occurred. A better understanding of the changes in the pulse shape will help to better locate and define the source of the lengthened prompt pulse for HYSPEC. This knowledge will help to characterize the changes in the energy spectrum of the prompt pulse caused by time of flight dispersion or material slowingdown affects from various materials at their respective locations in the immediate and distant vicinity.

It should be noted that this process was subject to the same constraints in data availability as the pulse magnitude portion of the analysis, meaning that of the full potential matrix of shutter and instrument configurations available to HYSPEC, only around half are actually represented in this analysis as there was no archived data available for the remaining shutter and instrument configuration combinations.

\subsection{STATISTICAL ANALYSIS OF DATA}

To quantify the fluctuations in magnitude using frequency distributions a statistical analysis was performed. For each HYSPEC configuration a mean of the total prompt pulse magnitudes was obtained $\left(\mu_{O(\#)}\right)$ from the frequency distributions with all shutters open $\left(P_{O(\#)}\right)$. Then the deviation of this distribution $\left(\sigma_{\mathrm{O}(\#)}\right)$ was calculated along with the uncertainty of the mean $\left(\Omega_{\mathrm{O}(\#)}\right)$. Then each individual shutter was analyzed being the only-one-closed $\left(P_{X X(\#)}\right)$ and the mean $\left(\mu_{X X(\#)}\right)$, deviation of the distribution $\left(\sigma_{X X(\#)}\right)$ and uncertainty in the mean was found $\left(\Omega_{\mathrm{XX}(\#)}\right)$ for each shutter. It should be noted that the analysis accounted for all beamline's primary shutters and secondary shutters where data was available. The difference between the two means $\left(\mu_{O(\#)}-\mu_{X X(\#)}=C_{X X(\#)}\right)$ represents the quantitative effect of each shutter. Details regarding the scripting and exact formulation of the statistical calculations can be found in Appendix I. Symbols and descriptions of these values can be found in Table 2. 
Table 2: Explanation of statistical symbols and terminology

Symbol

$\boldsymbol{P}_{\boldsymbol{o}(\#)}$

$\boldsymbol{\mu}_{\boldsymbol{O}(\#)}$

$\sigma_{0(\#)}$

$\boldsymbol{\Omega}_{\mathbf{0}(\#)}$

$\boldsymbol{P}_{X X(\#)}$

$\mu_{X X(\#)}$

$\sigma_{\mathbf{X X}(\#)}$

$\boldsymbol{\Omega}_{\mathbf{X X}(\#)}$

$C_{X X(\#)}$

$\sigma_{\mathrm{CXX}(\#)}$

$\boldsymbol{\Omega}_{\mathrm{CXX}(\#)}$

\section{Description}

$3 \mathrm{~ms}$ prompt pulse integration

$3 \mathrm{~ms}$ prompt pulse integration with all North-side shutters open and HYSPEC in configuration \#

Mean of all $P_{O(\#)}$ 's

Deviation of the distribution of $\mu_{O(\#)}$

Uncertainty of $\mu_{O(\#)}$

3ms prompt pulse integration with shutter $\mathrm{XX}$ being the only shutter closed and HYSPEC in configuration \#

Mean of all $P_{X X(\#)}$ 's

Width of the distribution of $\mu_{X X(\#)}$

Uncertainty of $\mu_{X X(\#)}$

Difference of the two means $\left(\mu_{O(\#)}-\mu_{X X(\#)}\right)$

Combined deviation of $\sigma_{\mathrm{O}(\#)}$ and $\sigma_{\mathrm{XX}(\#)}$

Combined uncertainty of $\Omega_{\mathrm{O}(\#)}$ and $\Omega_{\mathrm{XX}(\#)}$ 


\section{RESULTS}

The results produced in this analysis support previous investigations performed by the HYSPEC beamline staff. Also, new conclusive data regarding specific contributors to HYSPEC prompt pulse fluctuations are quantified within this section along with a more refined characterization of the SNS instrument hall's background signature as affected by various physical changes in the building.

\subsection{OBSERVATIONS}

Plotting the data over time revealed much about the behavior of the prompt pulse especially when fluctuations could be directly attributed to known external changes. One known external change to the target facility was the installation of new shielding blocks at a known "hot spot" around the shielding monolith around February $8^{\text {th }}, 2012$ [Figure 8]. This shielding installation took place during a regularly scheduled beam outage and the HYSPEC prompt pulse magnitude data can be seen to drop dramatically at dates that straddle this event. This agreement between known real-world changes and fluctuations reflected in the data support the accuracy of the methods used in this analysis.

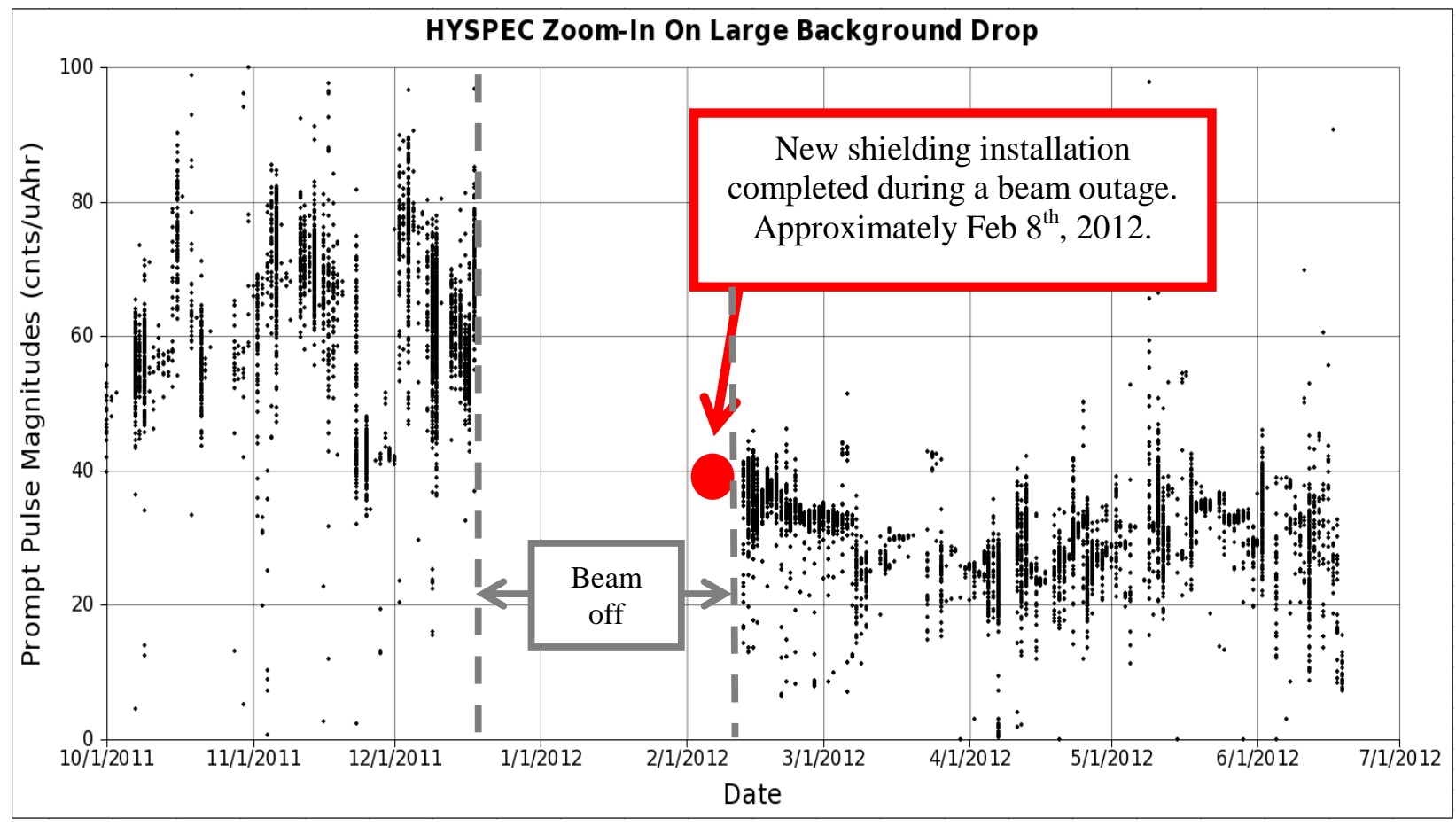

Figure 8: Drop in HYSPEC background after beam outage and shielding installation.

For further confirmation of the analysis process, direct correlations were found between shutters and prompt pulse magnitudes by coupling CSS data with the HYSPEC experimental data sets. In one specific situation, the prompt pulse magnitude was witnessed to fluctuate 
directly with beamline 13's secondary shutter. This can be seen in Figure 9 where the prompt pulse is directly affected by this shutter position by nearly $25 \%$.

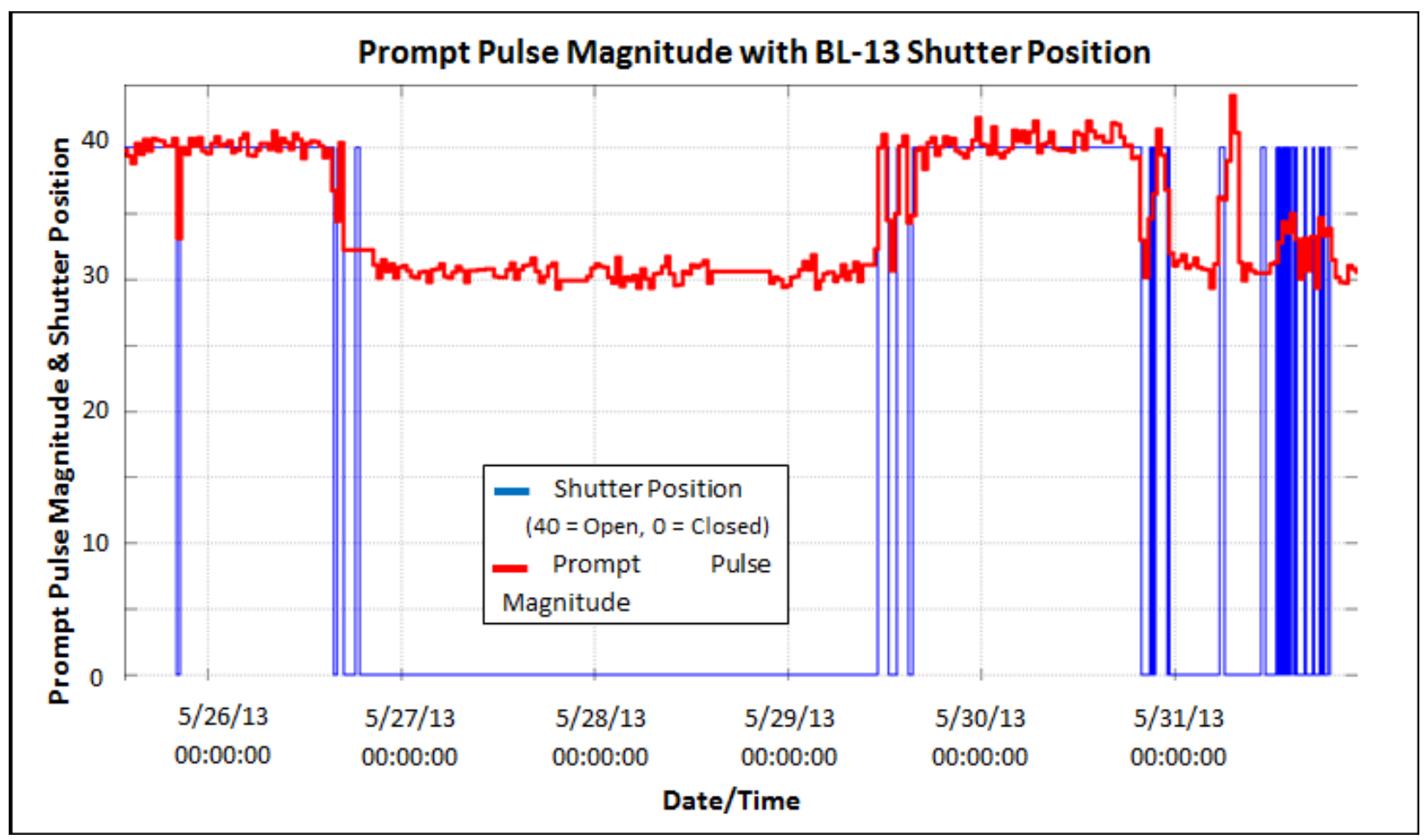

Figure 9: Beamline 13 shutter correlation with HYSPEC prompt pulse magnitude.

This observation further supported the accurate nature of the numerical data being produced and provided further confidence in the quantitative data being derived from this analysis.

\subsection{INTERNAL EFFECTS ON PROMPT PULSE MAGNITUDE}

Internal effects on the magnitude of the prompt pulse were confirmed and quantified with regard to HYSPEC's own configuration. Using data only from runs where all the shutters where open and finding the respective mean of each individual instrument configuration, a significant effect was observed that showed as the configuration of HYSPEC (see Figure 5) moved in a counter-clockwise fashion about the room, the prompt pulse signature diminished in magnitude.

Figure 10 illustrates this decrease in magnitude as a function of configuration and indicates that certain aspects of the local shielding geometry are important in understanding the effects of the prompt pulse around the detector room of HYSPEC. (The data series where points are missing indicate that HYSPEC was never in a certain configuration while that specific shutter was the only one closed). 


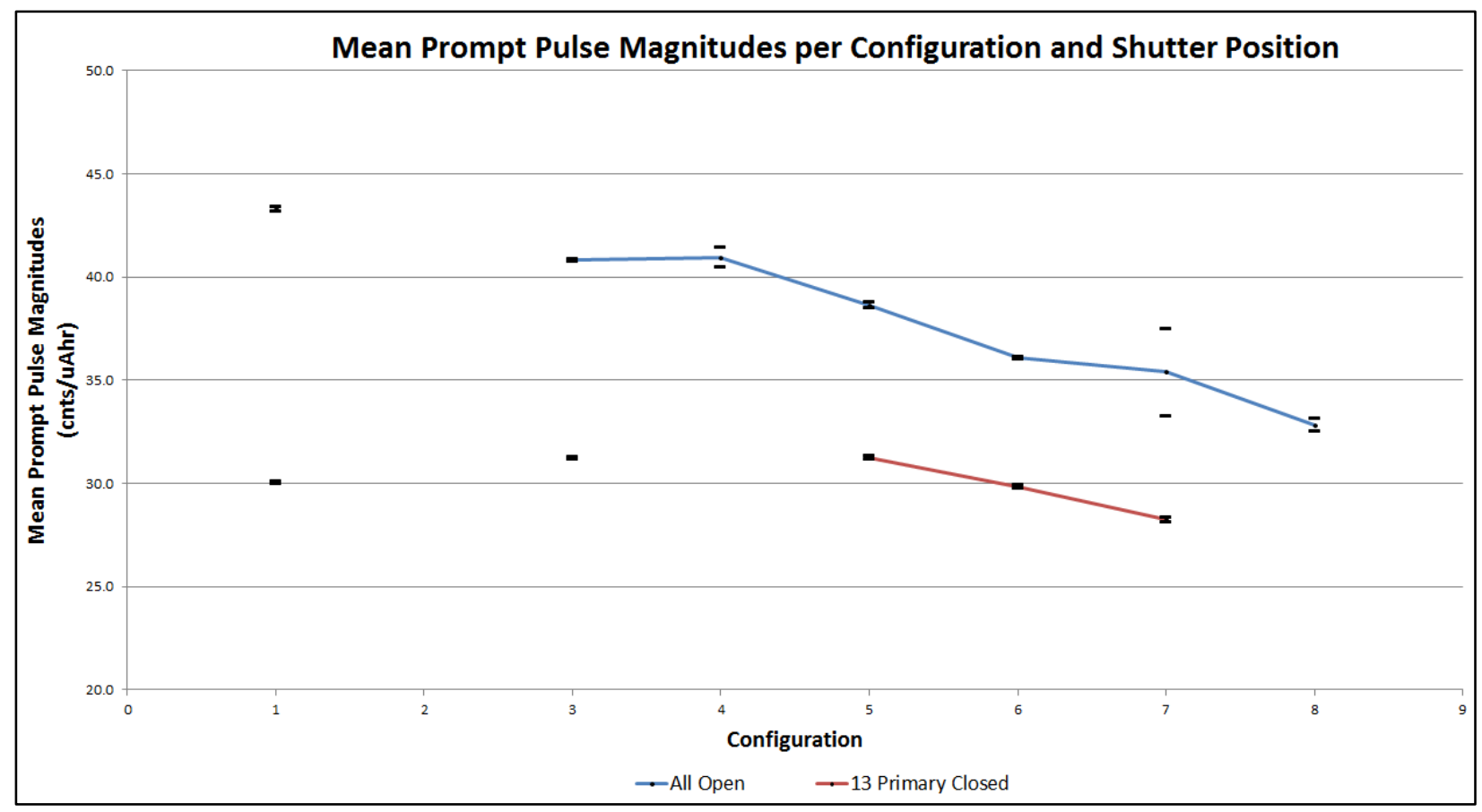

Figure 10: HYSPEC configurations and beamline 13 shutter effects. Error bars are in reference to the standard deviation of the mean for each respective value.

These configuration correlations were found to be beneficial in shedding light on the nature of where a portion of the prompt pulse is coming from. The results of this exercise show that under certain conditions there is a proximity relationship with one of their closest neighbors, beamline 13, by the fact that as HYSPEC moved away from this instrument while beamline 13's shutter was open, the background signature dropped significantly.

It should also be noted that this effect is only significant under specific conditions. When beamline 13 is open, HYSPEC's proximity to it (via configuration) greatly influences variations in the magnitude of the prompt pulse. This is illustrated in Figure 11 by the shifting of the peaks to the left on the $\mathrm{x}$-axis, signifying a decrease in prompt pulse magnitude as HYSPEC moves away from beamline 13. However, when beamline 13 is closed, configuration effects on the prompt pulse magnitude still exist but have less of a pronounced effect. This can be seen by the comparatively reduced slope of the "13 Primary Closed" series versus the "All Open" series on Figure 10 and also by the similar alignment along the $\mathrm{X}$-axis in Figure 12. These data imply that while other shutters do affect the background for HYSPEC, their effect is a broader one which is less affected by the configuration while the effects of beamline 13 are more acutely related to the spatial changes of HYSPEC. This is helpful knowledge in trying to diagnose the effects of local and non-local contributors to prompt pulse fluctuations for HYSPEC and is useful in regard to overall beamline design considerations along with beamstop, shutter and flightpath shielding strategies for any target facility with multiple beamlines in close proximity. 


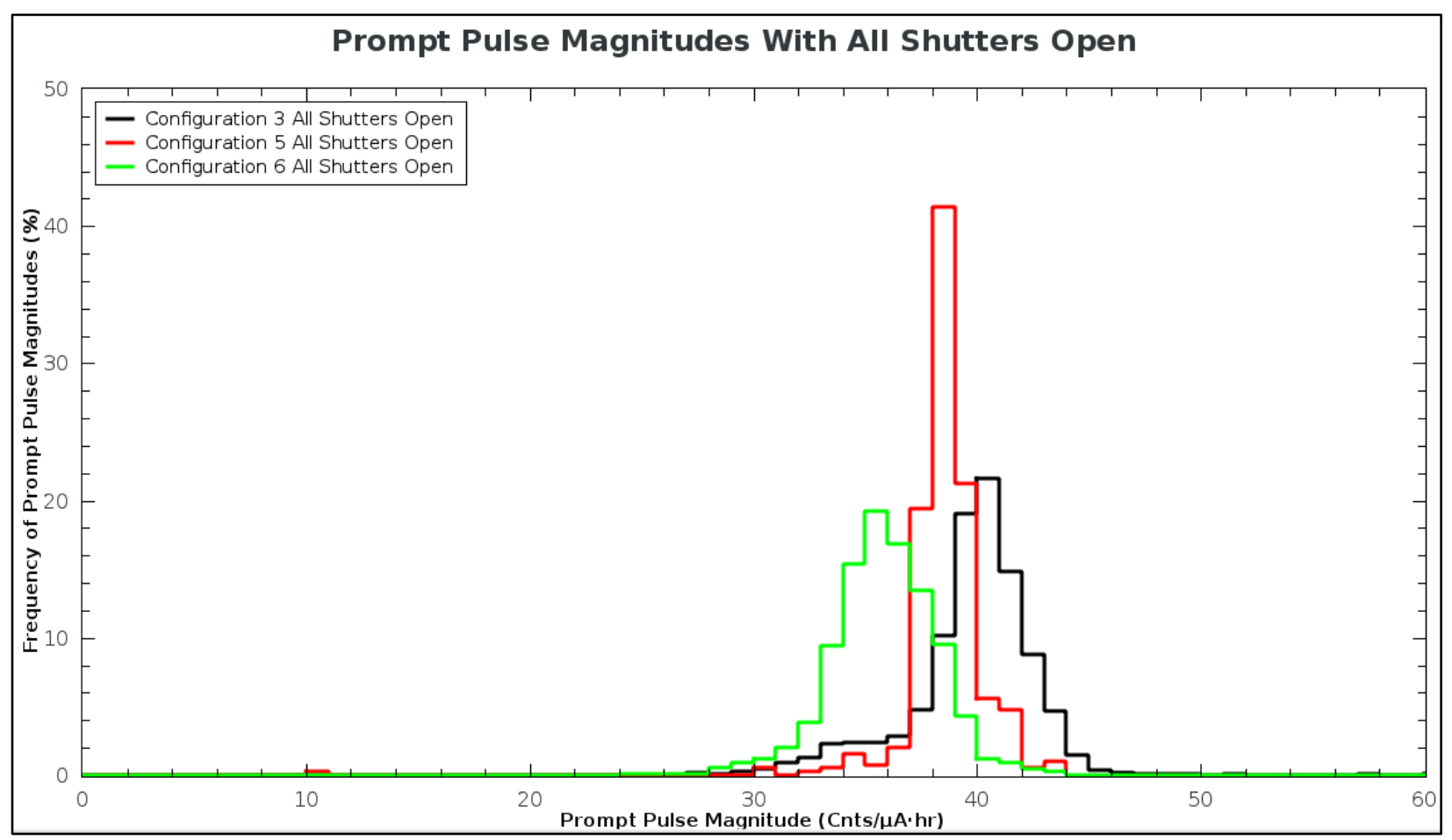

Figure 11: Large discrepancy between prompt pulse magnitudes clearly shifting as a function of configuration while BL13 shutter is open and all other North side shutters are open.

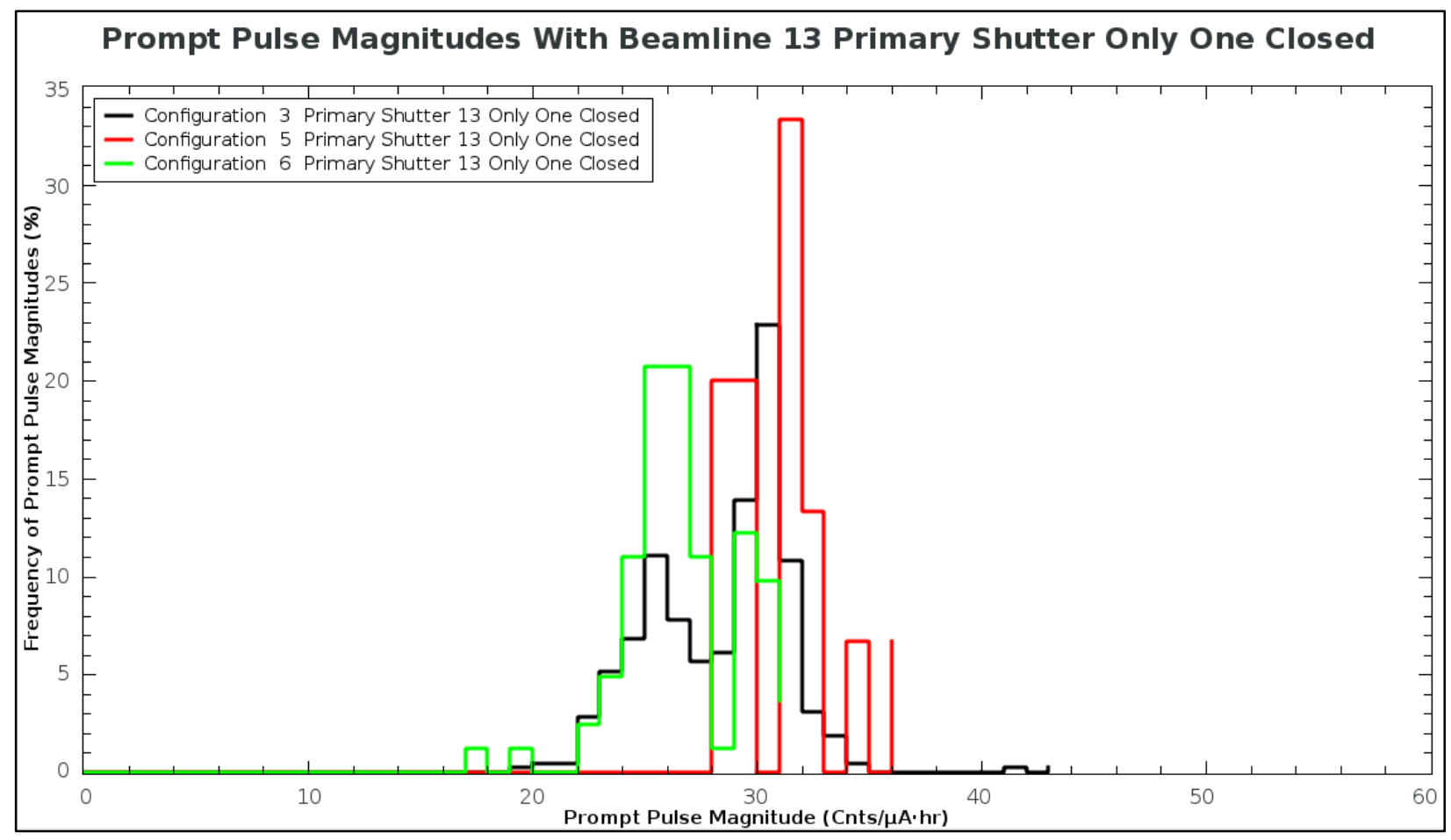

Figure 12: Prompt pulse magnitudes not clearly shifting as a function of configuration while BL13 shutter is closed and all other North side shutters are open. 
To further define the internal effects of HYSPEC, a run was conducted where HYSPEC's own shutter was closed while they were collecting data. This was done in an effort to determine if aspects of HYSPEC's own incident neutron beam played any role in the ambient background of their own detector room. This run was performed while all north-side shutters were open except for beamline 13 (and HYSPEC) and the instrument was in configuration 6 . The magnitude of the prompt pulse from this test run was found to be statistically equal in magnitude to the prompt pulse magnitudes of all other runs of the same instrument configuration and shutter arrangement but with HYPSEC shutters open. This indicates that HYSPEC's own shutter position, flight path and beam stop do not contribute to the changes in prompt pulse magnitude observed in this analysis.

The pulse shape as a function of HYSPEC instrument configuration was found to be a noticeable and repeated trend in the data. For instance, when 100 random runs from configuration 1 and configuration 3 where compared via Pearson-Chi Square tests with $\alpha=0.05$, the test static was found to be 77.4 with an associated $p$-value of 0.054 . This indicates that the differences seen in the two pulse shapes were most likely associated with statistical fluctuations and not an actual physical influence. This same test was performed on all configurations (configuration 2 had no available data) and the results are listed below in Table 3. Note that the critical Chi-Square test statistic for $\alpha=0.05$ is 79.1. Any Chi-Square above this critical value (red) represents a high likelyhood of the differences between the two distributions being due to factors other than normal statistical fluctuations and values below this critical value (black) repersent a high likelihood of the distributions being similar in shape and only different by virtue of natural statistical fluctuations.

Table 3: Pearson-Chi Square results for HYSPEC instrument configuration comparisons.

\begin{tabular}{lll}
\hline \hline Configurations Compared & Chi Square Test Statistic & Associated P-value \\
\hline Config. 1 VS Config. 3 & 77.4 & 0.054 \\
Config. 1 VS Config. 4 & 111.3 & $4.5 \mathrm{E}-5$ \\
Config. 1 VS Config. 5 & 84.8 & 0.015 \\
Config. 1 VS Config. 6 & 74.7 & 0.082 \\
Config. 1 VS Config. 7 & 100.6 & $6.0 \mathrm{E}-4$ \\
Config. 1 VS Config. 8 & 84.5 & 0.016 \\
\hline \hline
\end{tabular}

*For all comparisons, $\alpha=0.05$ and corresponding 'Critical Chi Square' $=79.1$.

Desite the statistical similarity and/or differences of these various configuration comparisons, all the comparisons had some level of dissimilarity. The actual arithmetic difference of the unitized pulse shapes was calculated to determine if there was any consistency to the location in time of these differences. It was found that there were consistant differences in TOF regions found between instrument configurations. These differences represent a change in the detected energy spectrum as a function of instrument configuration and can be used to identify and/or exclude potential candidates for sources of thermalization of the fast neutron background or a change in distance from a source location represented by a change in the time of flight dispersion from the source to HYSPEC relative to the distance between configurations. The differences of these particular comparisons can be seen in Figure 13 and the relative magnitudes are a result of configuration 1 minus the compared configuration. This means that, when there is a positive value in the early TOF region that configuration 1 sees a higher fast background profile then the compared configuration and the opposite is true for negative values. 
Again, this data doesn't directly implicate any particular source of thermalization or time of flight dispersion but does assist in the investigative process of better characterizing and understanding the circumstances governing changes in the background signal.

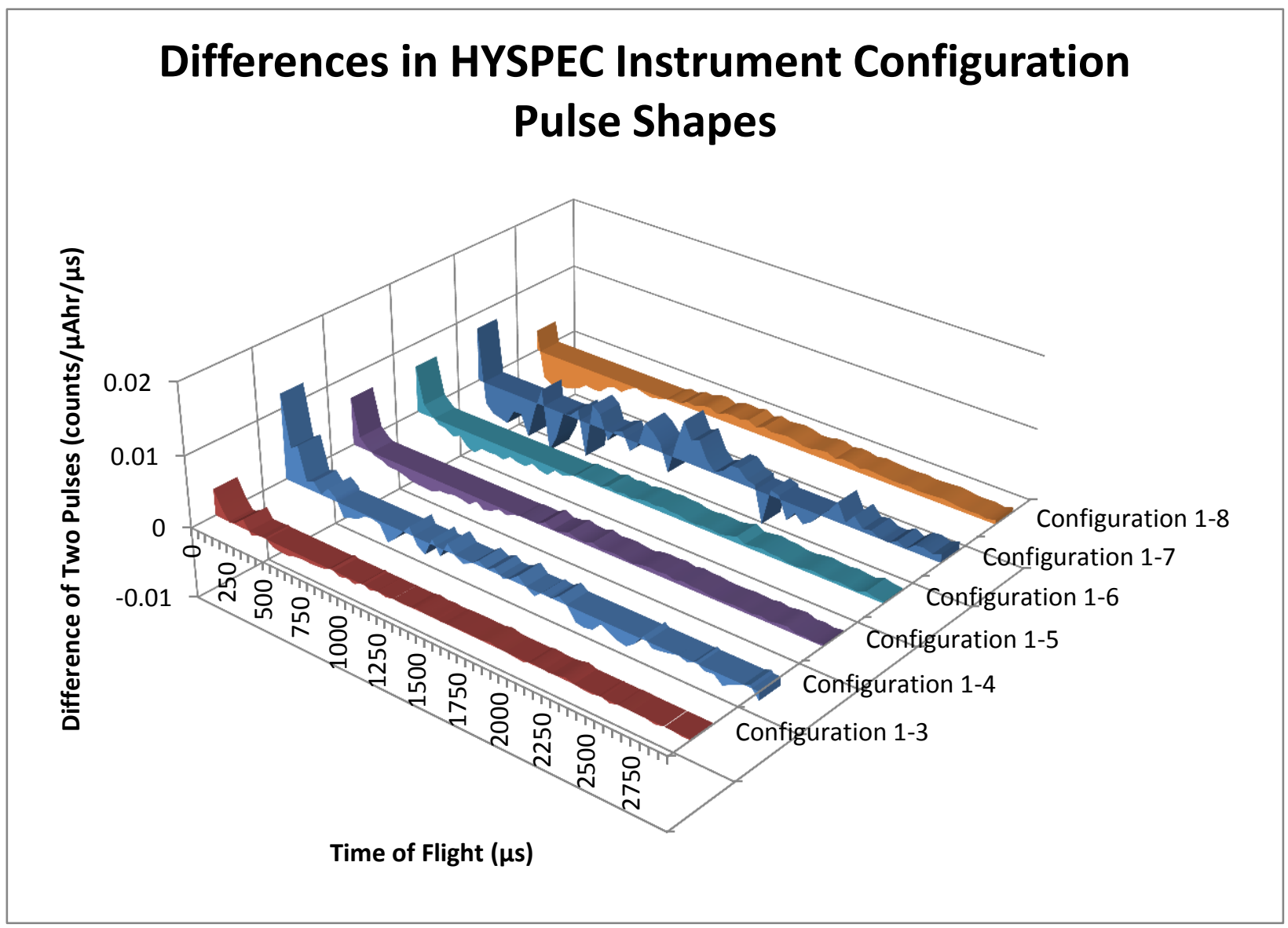

Figure 13: Arithmetic difference of pulse shapes for different instrument configurations. All configuration canonical pulse shapes are subtracted from configuration 1's canonical pulse shape. Configuration $\mathbf{2}$ had no available data sets for comparison. 


\subsection{EXTERNAL EFFECTS ON PROMPT PULSE MAGNITUDE}

The statistical analysis of the comparisons for each individual configuration along with each individual shutter's contribution proved to be enlightening as to where the majority of the causes of external variation in magnitude were coming from. These values are calculated by finding the difference of when all the shutters were open and when only one shutter was closed $\left(\mu_{O(\#)}-\mu_{X X(\#)}\right)$. The comprehensive data tables that produced the values listed in Table $4 \& 5$ can be found in Appendix I. It should be noted that in Tables 4 \& 5, " - " represents when there was no data available for that particular combination of parameters. Also, despite the statistical processes applied to achieve these numbers, values generated from data sets with less than five total runs are colored gray to signify an additional lack of confidence in these particular numbers [Table 4 \& 5].

Other specifics were discovered in this analysis by not just identifying if there were fluctuations in magnitude caused by a shutter position, but whether these fluctuations contributed positively or negatively to the overall background magnitude. For instance, typically the background signature drops when a neighboring shutter is closed but this analysis revealed, in certain configurations for HYSPEC, there are specific shutters that cause a rise in prompt pulse magnitudes when closed. To help the reader quickly differentiate between these positive and negative effects on the prompt pulse magnitude, each value in Table $4 \& 5$ is followed by a ( $\uparrow)$, $(\downarrow)$ or $(=)$ sign to indicate whether the particular shutter being closed; increases, decreases or doesn't affect the background magnitude. This relationship is important to understand because if a shutter being closed increases the ambient background, it implies a shielding inadequacy in the vicinity of the shutter in question as opposed to the beamstop at the end of the flight path being the source of secondary neutron scattering. 
Table 4: HYSPEC background signature while specific shutter is the only shutter closed on the north side of the target facility. All values are cnts/ $\mu$ Ahr (Exact uncertainties can be found in Appendix I).

\begin{tabular}{|c|c|c|c|c|c|c|c|c|}
\hline $\begin{array}{l}\text { Beamline } \\
\text { shutter }\end{array}$ & $\begin{array}{l}\text { Config } \\
1\end{array}$ & $\begin{array}{l}\text { Config } \\
2\end{array}$ & $\begin{array}{l}\text { Config } \\
3\end{array}$ & $\begin{array}{l}\text { Config } \\
4\end{array}$ & $\begin{array}{l}\text { Config } \\
5\end{array}$ & $\begin{array}{l}\text { Config } \\
6\end{array}$ & $\begin{array}{l}\text { Config } \\
7\end{array}$ & $\begin{array}{l}\text { Config } \\
8\end{array}$ \\
\hline $\begin{array}{c}\mu_{\boldsymbol{O}(\#)} \\
\text { All Open }\end{array}$ & 43.3 & 0.0 & 40.8 & 41.0 & 38.7 & 36.1 & 35.4 & 32.8 \\
\hline $\begin{array}{c}\mu_{11(\#)} \\
\text { Primary }\end{array}$ & - & - & - & - & - & - & - & - \\
\hline $\begin{array}{c}\mu_{11 a(\#)} \\
\text { Secondary }\end{array}$ & $43.6 \uparrow$ & - & $40.7 \downarrow$ & $34.2 \downarrow$ & $38.2 \downarrow$ & $36.6 \downarrow$ & - & - \\
\hline $\begin{array}{c}\mu_{11 b(\#)} \\
\text { Secondary }\end{array}$ & - & - & $39.7 \downarrow$ & - & - & $34.7 \downarrow$ & - & - \\
\hline $\begin{array}{c}\mu_{12(\#)} \\
\text { Primary }\end{array}$ & - & - & $34.6 \downarrow$ & - & - & $36.1=$ & - & $30.8 \downarrow$ \\
\hline $\begin{array}{c}\mu_{13(\#)} \\
\text { Primary }\end{array}$ & $30.0 \downarrow$ & - & $31.3 \downarrow$ & - & $31.2 \downarrow$ & $29.8 \downarrow$ & $28.2 \downarrow$ & - \\
\hline $\begin{array}{c}\mu_{13(\#)} \\
\text { Secondary }\end{array}$ & $32.5 \downarrow$ & - & $31.9 \downarrow$ & - & $31.4 \downarrow$ & $28.3 \downarrow$ & $29.6 \downarrow$ & - \\
\hline $\begin{array}{c}\mu_{15(\#)} \\
\text { Primary }\end{array}$ & - & - & - & - & - & - & - & - \\
\hline $\begin{array}{c}\mu_{15(\#)} \\
\text { Secondary }\end{array}$ & $45.2 \uparrow$ & - & $40.1 \downarrow$ & - & $38.2 \downarrow$ & $34.6 \downarrow$ & - & $33.4 \uparrow$ \\
\hline $\begin{array}{c}\mu_{16(\#)} \\
\text { Primary }\end{array}$ & - & - & $41.5 \uparrow$ & - & - & $37.5 \uparrow$ & - & $34.0 \uparrow$ \\
\hline $\begin{array}{c}\mu_{16(\#)} \\
\text { Secondary }\end{array}$ & $42.0 \downarrow$ & - & $39.5 \downarrow$ & - & $39.0 \uparrow$ & $36.3 \uparrow$ & $38.9 \uparrow$ & $35.2 \uparrow$ \\
\hline $\begin{array}{c}\mu_{17(\#)} \\
\text { Primary }\end{array}$ & $41.1 \downarrow$ & - & $39.9 \downarrow$ & - & $37.7 \downarrow$ & $35.7 \downarrow$ & - & $33.6 \uparrow$ \\
\hline $\begin{array}{c}\mu_{18(\#)} \\
\text { Primary }\end{array}$ & $48.5 \uparrow$ & - & $40.2 \downarrow$ & - & $38.1 \downarrow$ & $37.4 \uparrow$ & - & $40.3 \uparrow$ \\
\hline
\end{tabular}


While the data on the previous table displayed the total background magnitude with individual shutters in a particular position, the following table shows the mean of all prompt pulse magnitudes while all shutters were open minus the mean of all prompt pulse magnitudes while a shutter was the only one closed ( $\left.\boldsymbol{\mu}_{\boldsymbol{O}(\#)}-\boldsymbol{\mu}_{\boldsymbol{X}(\#)}\right)$ thus providing the contribution of each individual shutter [Table 5].

Table 5: Difference in prompt pulse magnitude while the specific shutter is the only one closed and when all the shutters are open. All values are cnts/ $\mu \mathrm{Ahr}$ (Exact uncertainties can be found in Appendix I).

\begin{tabular}{|c|c|c|c|c|c|c|c|c|}
\hline $\begin{array}{l}\text { Beamline } \\
\text { shutter }\end{array}$ & $\begin{array}{l}\text { Config } \\
1\end{array}$ & $\begin{array}{l}\text { Config } \\
2\end{array}$ & $\begin{array}{l}\text { Config } \\
3\end{array}$ & $\begin{array}{l}\text { Config } \\
4\end{array}$ & $\begin{array}{l}\text { Config } \\
5\end{array}$ & $\begin{array}{l}\text { Config } \\
6\end{array}$ & $\begin{array}{l}\text { Config } \\
7\end{array}$ & $\begin{array}{l}\text { Config } \\
8\end{array}$ \\
\hline $\begin{array}{r}\mu_{o(\#)} \\
\text { All Open }\end{array}$ & 43.3 & 0.0 & 40.8 & 41.0 & 38.7 & 36.1 & 35.4 & 32.8 \\
\hline $\begin{array}{c}C_{11(\#)} \\
\text { Primary }\end{array}$ & - & - & - & - & - & - & - & - \\
\hline $\begin{array}{c}C_{11 a(\#)} \\
\text { Secondary }\end{array}$ & $0.3 \uparrow$ & - & $0.1 \downarrow$ & $6.8 \downarrow$ & $0.5 \downarrow$ & $0.5 \downarrow$ & - & - \\
\hline $\begin{array}{c}C_{11 b(\#)} \\
\text { Secondary }\end{array}$ & - & - & $1.1 \downarrow$ & - & - & $1.4 \downarrow$ & - & - \\
\hline $\begin{array}{c}C_{12(\#)} \\
\text { Primary }\end{array}$ & - & - & $6.2 \downarrow$ & - & - & 0.0 & - & $2.0 \downarrow$ \\
\hline $\begin{array}{c}C_{13(\#)} \\
\text { Primary }\end{array}$ & $13.3 \downarrow$ & - & $9.5 \downarrow$ & - & $7.5 \downarrow$ & $6.3 \downarrow$ & $7.2 \downarrow$ & - \\
\hline $\begin{array}{c}C_{13(\#)} \\
\text { Secondary }\end{array}$ & $10.8 \downarrow$ & - & $8.9 \downarrow$ & - & $7.3 \downarrow$ & $7.8 \downarrow$ & $5.8 \downarrow$ & - \\
\hline $\begin{array}{c}C_{15(\#)} \\
\text { Primary }\end{array}$ & - & - & - & - & - & - & - & - \\
\hline $\begin{array}{c}C_{15(\#)} \\
\text { Secondary }\end{array}$ & $1.9 \uparrow$ & - & $0.7 \downarrow$ & - & $0.5 \downarrow$ & $1.5 \downarrow$ & - & $0.6 \uparrow$ \\
\hline $\begin{array}{c}C_{16(\#)} \\
\text { Primary }\end{array}$ & - & - & $0.7 \uparrow$ & - & - & $1.4 \uparrow$ & - & $1.2 \uparrow$ \\
\hline $\begin{array}{c}C_{16(\#)} \\
\text { Secondary }\end{array}$ & $1.3 \downarrow$ & - & $1.3 \downarrow$ & - & $0.3 \uparrow$ & $0.2 \uparrow$ & $3.5 \uparrow$ & $2.4 \uparrow$ \\
\hline $\begin{array}{c}C_{17(\#)} \\
\text { Primary }\end{array}$ & $2.2 \downarrow$ & - & $0.9 \downarrow$ & - & $1.0 \downarrow$ & $0.4 \downarrow$ & - & $0.8 \uparrow$ \\
\hline $\begin{array}{c}C_{18(\#)} \\
\text { Primary }\end{array}$ & $5.2 \uparrow$ & - & $0.6 \downarrow$ & - & $0.6 \downarrow$ & $1.3 \uparrow$ & - & $7.5 \uparrow$ \\
\hline $\begin{array}{l}\mu_{O(\#)}-\Sigma C_{X X(\#)} \\
\text { Everything } \\
\text { Else }\end{array}$ & 19.1 & - & 20.4 & - & 28.8 & 23.0 & 28.2 & 27.5 \\
\hline
\end{tabular}


It should be mentioned that the "Everything Else" values are calculated by summing the collective contributions of all beamlines for a given HYSPEC configuration [Table 5]. Despite this general approach, beamlines consisting of a primary and secondary shutter had to be assessed as a "worst case scenario" type of method. Meaning, the counts $\mu \mathrm{A} \cdot \mathrm{hr}$ due to a beamline's primary shutter being closed cannot be assumed as totally independent from the counts $\mu \mathrm{A} \cdot \mathrm{hr}$ due to a beamline's secondary shutter being closed. Because of this, on beamlines where there are multiple shutters, the highest contributor (be it primary or secondary) is the one used to find the "Everything Else" values.

Also, before conclusive statements could be made on the "Everything Else" portion of the prompt pulse, the underrepresented beamline shutters needed to be generally characterized to make sure that they weren't a gross contributor. Since the computerized analysis required a beamline to be the only shutter open in order to be assessed, a limitation was imposed on available shutter scenarios. The result of this stipulation was that no data was available for beamline 11's and 15's primary shutters. To address the general contribution of these shutters a hand analysis was performed for each. A period of time was identified for beamline 11 and 15 (separately) where they were not the only one closed, but one of a few closed. During this time period, the contributions of all the other closed shutters were known and the sum of these various contributions could be subtracted from the "All Open" value for a given configuration. The observed mean of HYSPEC's prompt pulse during this period was then compared to this difference. When compared, beamline 11's primary shutter was found to contribute 0.1 counts $\mu \mathrm{A} \cdot \mathrm{hr}$ and beamline 15 's primary shutter was found to have 0.7 counts $\mu \mathrm{A} \cdot \mathrm{hr}$, both of which are negligible enough to feel confident of the "Everything Else" values being relatively accurate.

To further illustrate the overall impact of beamline contributions, an average of each beamline's contribution across all configurations was calculated. It should be noted that this average value only included data points with a statistical significance. For example, In Table 5, row $3\left(C_{11 a(\#)}\right)$, the average was calculated from the values 0.3 ("Config 1"), 0.1 ("Config 3") and 0.5 ("Config 6") only, not including zeros for the un-represented configurations or the "grayed out", statistically insignificant values. Also, since this approach addresses a total beamline impact, not differentiating between primary and secondary shutters, the "worst case scenario" for each beamline was used in the calculations. For example: The average impact of closing beamline 13's primary shutter (across all configurations) was found to account for a decrease of 8.8 counts $/ \mu \mathrm{A} \cdot \mathrm{hr}$, while the secondary shutter accounted for a decrease of 8.1 counts $\mu \mathrm{A} \cdot \mathrm{hr}$. Seeing as the primary shutter being closed accounted for a larger reduction in prompt pulse magnitude than the secondary, and thus a higher increase in magnitude when open, the primary was used to represent the "worst case scenario" of potential shutter configurations for this particular beamline. The values of the prompt pulses averaged across all configurations for each beamline along with the average when all shutters are open are shown in Table 6. 
Table 6: Average portion of the prompt pulse magnitude across all HYSPEC configurations for all north side beamlines. All values are cnts/ $\mu \mathrm{Ahr}$

\begin{tabular}{llllllllll}
\hline $\begin{array}{l}\text { All } \\
\text { Open }\end{array}$ & BL11-a & BL-11b & BL-12 & BL-13 & BL-15 & BL-16 & BL-17 & BL-18 & $\begin{array}{l}\text { Everything } \\
\text { Else }\end{array}$ \\
\hline 33.5 & 0.2 & 1.3 & 4.2 & 9.0 & 1.0 & 1.7 & 1.0 & 1.0 & 13.1 \\
\hline \hline
\end{tabular}

Using the data in Table 6, a chart was made to further illustrate the relative impact of each neighboring beamline on the prompt pulse along with the percentage of unaccounted-for portions of the prompt pulse as seen by HYSPEC [Figure 14].

\section{Contributions to HYSPEC Prompt Pulse}

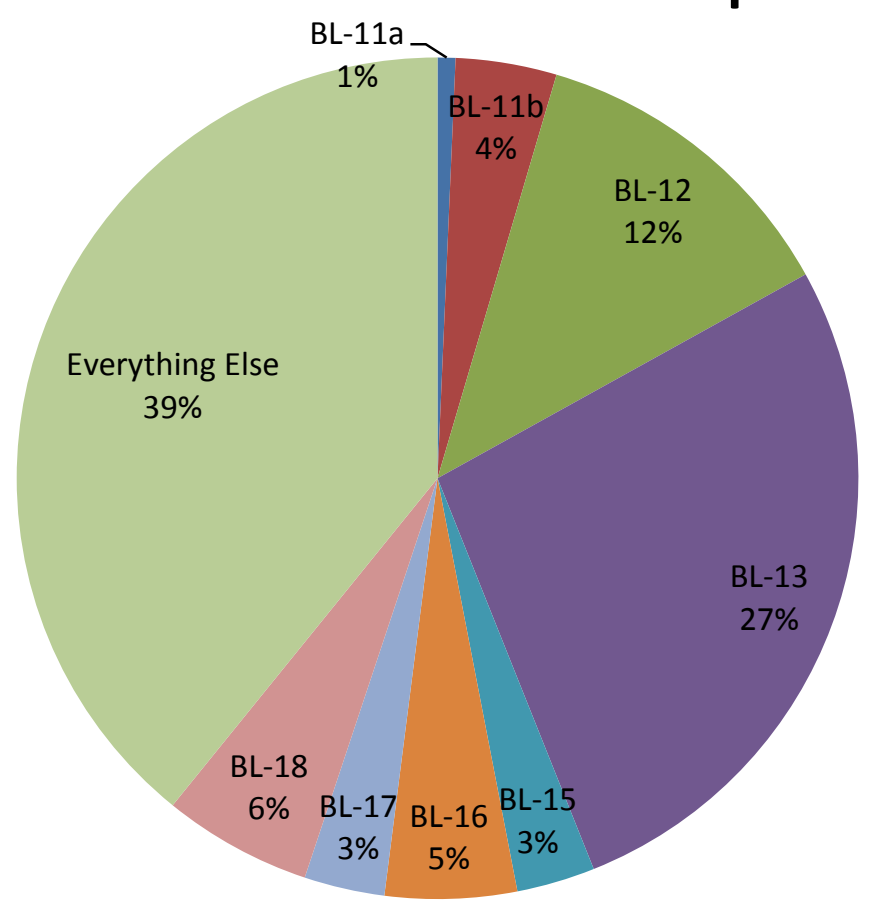

Figure 14: Contributions to HYSPEC prompt pulse magnitude as a percentage of average pulse when all shutters are open. Total pulse magnitude is approximately 33 counts $/ \mu \mathrm{A} \cdot \mathrm{hr}$.

It should be noted that with the available data, the overall contributions of neighboring shutters accounts for approximately $61 \%$ of the observable prompt pulse magnitude for HYSPEC. Again, it should be noted that this is a generalization of the observed contributors to the prompt pulse background.

At first glance, it seems that by eliminating all outside contributors to the prompt pulse (i.e. adding shielding to specific points along neighboring beamlines) that the prompt pulse could be decreased by $61 \%$ for HYSPEC. Although this could be true, a cursory look at how this would ultimately affect the data collection time of HYSPEC will now be presented. To help understand what eliminating these external contributing factors might mean for HYSPEC, a plot of a typical HYSPEC user data file (focused on the prompt pulse) is plotted along with the identical spectrum artificially reduced by $61 \%$ [Figure 15]. 


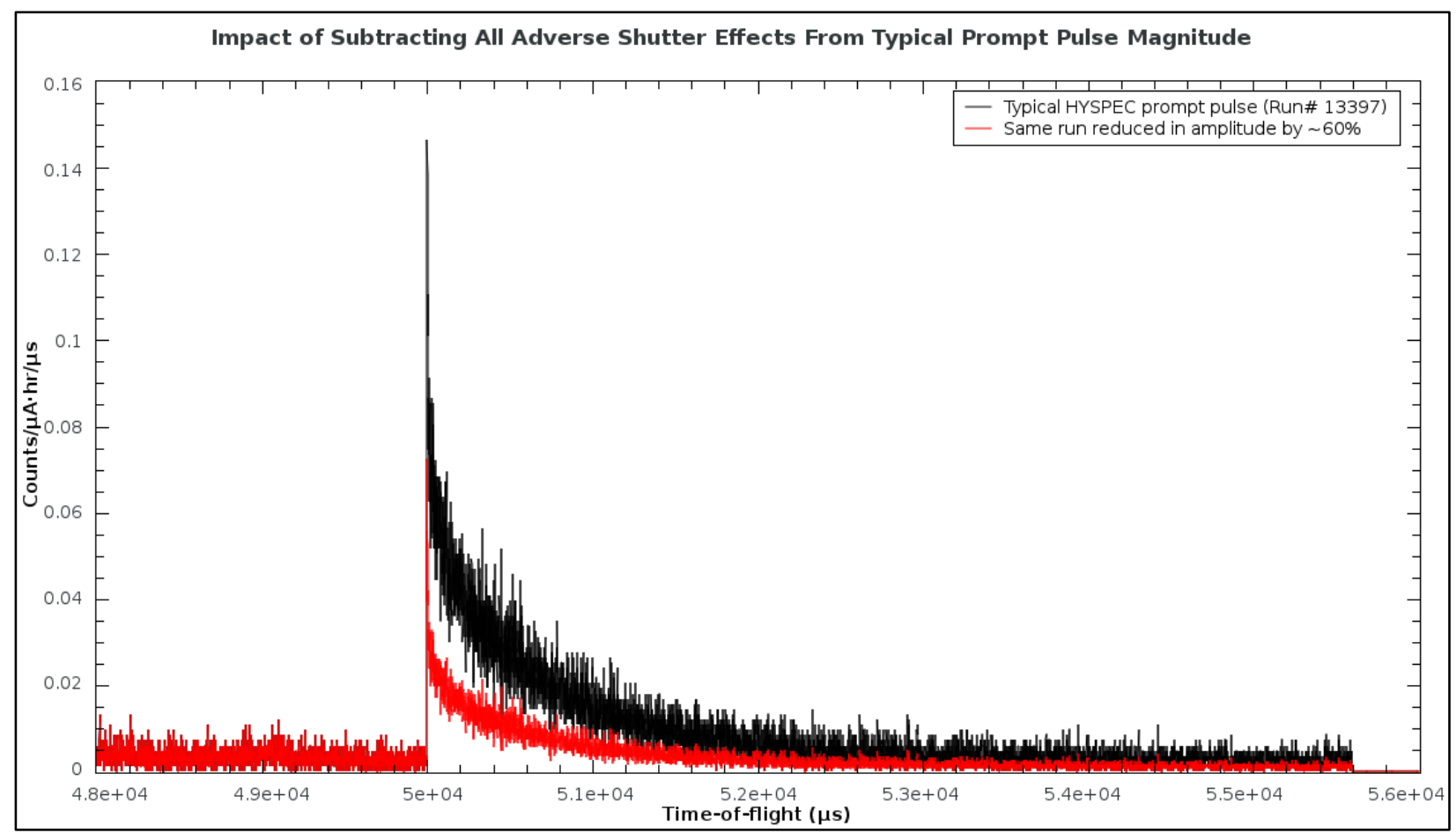

Figure 15: Hypothetical prompt pulse with all identified contributors eliminated (red) compared to a typical prompt pulse with all shutters in their maximum adverse configuration (black).

What's important to note here is that while the peak magnitude is noticed to decrease significantly, the tail of the pulse still occupies over $2.0 \mathrm{~ms}$ of potential time-of-flight data and any TOF region occupied by the prompt pulse is considered insufficient for users to collect data within. Even if a major shielding effort were to take place for all neighboring beamlines and all external observable contributors to HYSPEC's background were mitigated, a decrease in magnitude of $60 \%$ only results in a gain of potential TOF window of around $33 \%$ at best (or approximately $1.0 \mathrm{~ms}$ ).

The shape of the pulse was also observed to change as a function of shutter configuration. Canonical pulse shapes were determined for shutter configurations in the same method as the instrument configuration canonical pulses in that the shapes were derived from randomly selecting 100 runs (if available) for each shutter configuration and averaging each time bin of the pulse over all 100 runs. These canonical pulses could then be compared to one another by taking a difference. Similar to the results of the pulse magnitude comparisons, the shape seemed to be noticeably affected by some beamline's shutter positions and unnoticeable by other beamline shutter positions. Further analysis of these observed variations from one shutter configuration to the next will be pursued, but is beyond the scope of this analysis.

Figure 16 shows two examples comparing when all shutters are open versus when a specific shutter is the only one closed. Change in pulse shape from shutter to shutter is hypothesized to be caused by a time of flight dispersion due to differences in distance from HYSPEC to the beamline shutter being analyzed. Although this hypothesis is logical, no exact calculations have been performed as yet to define an exact location with this data. Further investigation of this hypothesis will continue. 


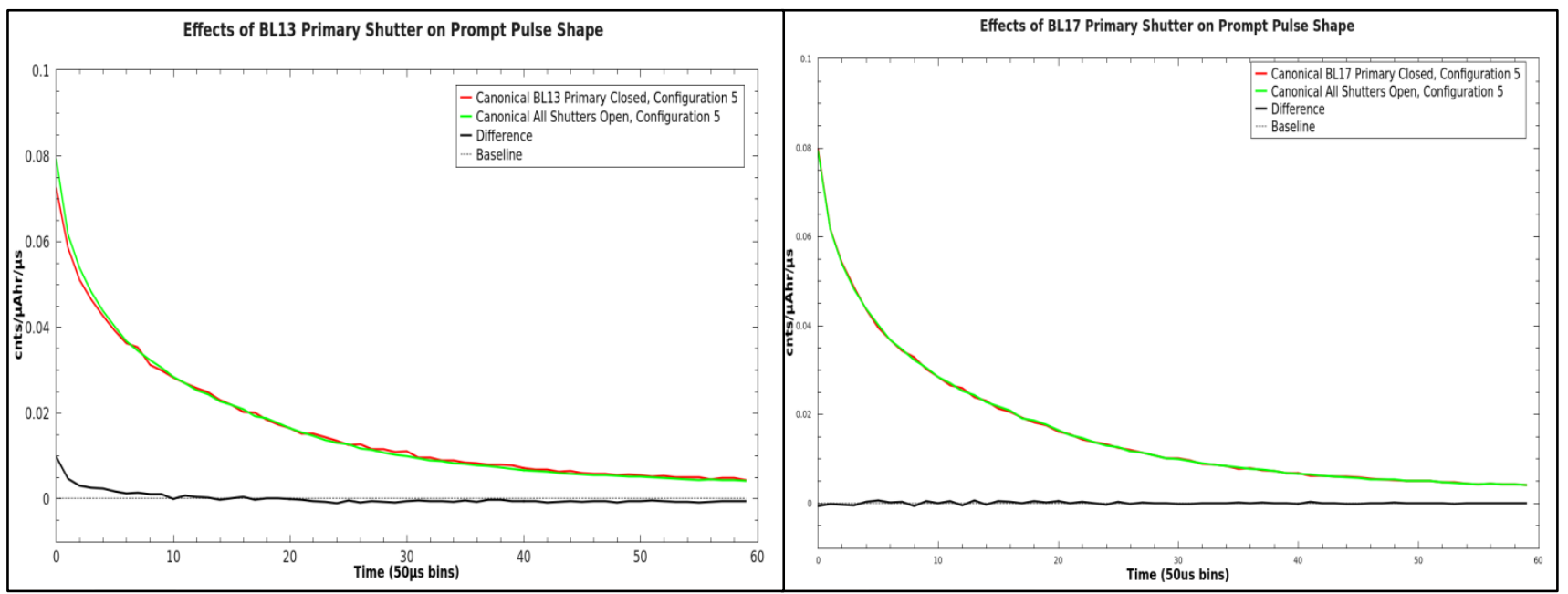

Figure 16: Beamline shutter contributing to prompt pulse shape change (left). Beamline shutter not contributing to prompt pulse shape change (right). 
Page $\mathbf{2 4}$ of $\mathbf{3 0}$ 


\section{CONCLUSIONS}

The purpose of this investigation was to identify local (within HYSPEC outbuilding) and non-local (outside HYSPEC outbuilding) contributors to prompt pulse fluctuations as detected by HYSPEC. This analysis provided quantitative and qualitative insight into specific causes of variations in prompt pulse magnitudes. It also helped to better classify the relative contributions of internal and external factors to the overall background signature within the SNS target building.

This analysis provides quantitative data showing that beamline shutter positions can have either a favorable or unfavorable effect on HYSPEC's reception of the magnitude of the prompt pulse. This improved assessment adds to the understanding of cause-and-effect relationships between background fluctuations and specific physical changes in the target building, and is valuable for understanding how to best monitor, manage and hopefully mitigate the instrument hall's background levels for HYSPEC and other instruments. The configuration of the HYSPEC detector vessel in the room was shown to influence both the magnitude and shape of the prompt pulse, although HYSPEC's own shutter position, flight path and beam stop did not appreciably contribute to changes in prompt pulse magnitudes observed in the analysis.

The changes in prompt pulse shape due to shutter configuration along with changes noticed in shape due to HYSPEC configuration implies that a change in the time of flight dispersion phenomenon is directly affected by the location or distance of the detector vessel from various predominant sources of pulsed background. The trends of pulse shape fluctuations observed in this analysis may help support efforts to identify exact locations of prompt pulse contributors by methods of triangulation and kinematic modeling.

During the course of this analysis, multiple shutter and instrument configurations were studied and representative pulse shapes and magnitudes were constructed for each available set of circumstances. These representative or canonical pulse shapes were used to distinguish differences in pulse magnitude and shape but were experimented with as a means of timedependent background subtractions. Meaning, if a user wanted to conduct a run at HYSPEC with an incident neutron energy that corresponded to a TOF window occupied by the prompt pulse, the canonical pulse representing the user's particular experimental instrument set-up and incidental shutter configuration could potentially be subtracted from the data set after the data had been collected. This method has only been loosely experimented with but showed promising results and would most likely only serve as an alternative solution to the problem since identifying and removing the prompt pulse all together for HYSPEC is the preferred solution.

Perhaps the largest take-away from this analysis is the fact that even when all shutters are in their optimum position and HYSPEC is in its optimum configuration for reducing prompt pulse magnitudes, there is still $\sim 40 \%$ of the total prompt pulse magnitude present and approximately $66 \%$ of the TOF region occupied by the best-case-scenario pulse still remains. The decision to pursue the shielding of neighboring beamlines versus the local shielding of HYSPEC is not within the scope of this analysis but the data contained within should help to make an educated decision on which avenue is most prudent to pursue. 



\section{REFERENCES}

1. Recent progress on HYSPEC, and its polarization analysis capabilities

Barry Winn, Uwe Filges, V. Ovidiu Garlea, Melissa Graves-Brook, Mark Hagen, Chenyang Jiang, Michel Kenzelmann, Larry Passell, Stephen M. Shapiro, Xin Tong, Igor Zaliznyak

EPJ Web of Conferences 8303017 (2015), DOI: 10.1051/epjconf/20158303017

2. Mantid (2013): Manipulation and Analysis Toolkit for Instrument Data.; Mantid Project. http://dx.doi.org/10.5286/SOFTWARE/MANTID

3. Python: http://www.python.org/

4. Control Systems Studio (CSS): http://controlsystemstudio.org/ 



\section{APPENDIX A. RUN DURATION AND CUMULATIVE CHARGE FILTER}

The duration of a given HYSPEC experiment can range from seconds to days depending on the experiment the users were performing. Also, a particular experiment could have taken place for a longer duration but coincidentally had lower beam intensity for a portion of the run. This scenario would yield a lower cumulative charge for the run which results in an artificially low average beam power for the run as well. Either of these aspects, or the combination of both, creates inconsistencies in the statistical density of the data sets and had to be considered in the qualification process as features of the analysis's algorithms focused on small TOF bins which were highly subject to yielding erroneous results from runs with small neutron counts.

The original solution was to declare that the overall run's charge accumulation needed to exceed some minimum threshold that one would instinctively set very high to render only the most densely populated data sets. It was quickly noticed that a balance was required between compromising high statistics on a per run basis versus having enough qualifying runs to yield good statistics for the overall analysis. A compromise was decided on at a minimum of 0.1 cumulative Coulombs per run as the threshold for filtering and the qualification of this parameter yielded both densely populated individual data sets and a sufficient number of runs. This number was found by accessing the "proton_charge" data of the run's log information which consisted of arrays containing the number of incident protons on the target and their respective columbic charge upon impact. Individual values in this array represent the charge of a single proton impact on the target. Values that contained no charge (or extremely low charge) were eliminated and an overall charge average was determined from all the recorded impacts. This average was multiplied by the number of individual impacts per run and an over-all average cumulative charge was found. If the total cumulative charge was less than 0.1 Coulombs than the run was found to have insufficient statistics for this analysis and thus excluded. It should be reiterated that this criterion was determined by a trial and error process while trying to balance the overall number of runs with individual run statistics. Future efforts to further this analysis could endeavor to find more sophisticated methods of filtration that would produce less disqualified runs and thus produce higher statistics.

The ultimate result of the parameters chosen for this filter was that approximately 18,671 runs were excluded from the final analysis. 



\section{APPENDIX B. DETECTOR VESSEL LOCATION FILTER}

As a means to utilize multiple wavelengths of neutrons, HYSPEC relies on Bragg scattering techniques which require the detector vessel to change position depending on the incident energy of neutron requested by the user. The instrument has two pivot axes, one about the monochromating crystal ( $\mathrm{m} 1$ ) and one about the sample (s2). The decision was made that the location of the detector vessel in the room is a variable that needed to be monitored during this analysis. Each run has in its log information, the position(s) of the detector vessel at the time of data collection. This analysis desired to not only know where the detector was in the room but also if it moved during data collection (which is not unheard of for this instrument). Since the purpose of classifying the location of the detector vessel was to isolate a run variable, runs that took place while the machine was in motion were disqualified. This was simply done by accessing the $\log$ information and observing the positions of $\mathrm{m} 1$ and s2. If either of these variables had more than one value in their array (representing more than one position) the run was disqualified.

Given the physical relationship of the detector vessel, detector room and the pivot angles, the limitations for possible regions and orientations of the detector can be geometrically predicted. These limitations are important when determining if a user can physically obtain the instrument configuration that their experiment asks for. For a check in this determination, a simple Python plotting program is supplied to users by the beamline staff so they may see if the machine is physically capable of achieving the position they desire for their experiment [Figure 17 and 18]. From the parameters in this program, extreme physical positions can be set as criteria for excluding a run in the analysis. This may seem redundant as one would assume that if a machine cannot physically occupy a certain location (because of a wall or some other obstruction) that it simply would never be there and thus the log data would not contain a run where this had occurred. This would be true execept it was found that in the early stages of the machines history, erroneous data was discovered in the position information and this needed to be addressed to have the analysis contain only accurate instrument position data. After the limiting parameters were set in place for the position filter approximately 400 runs were
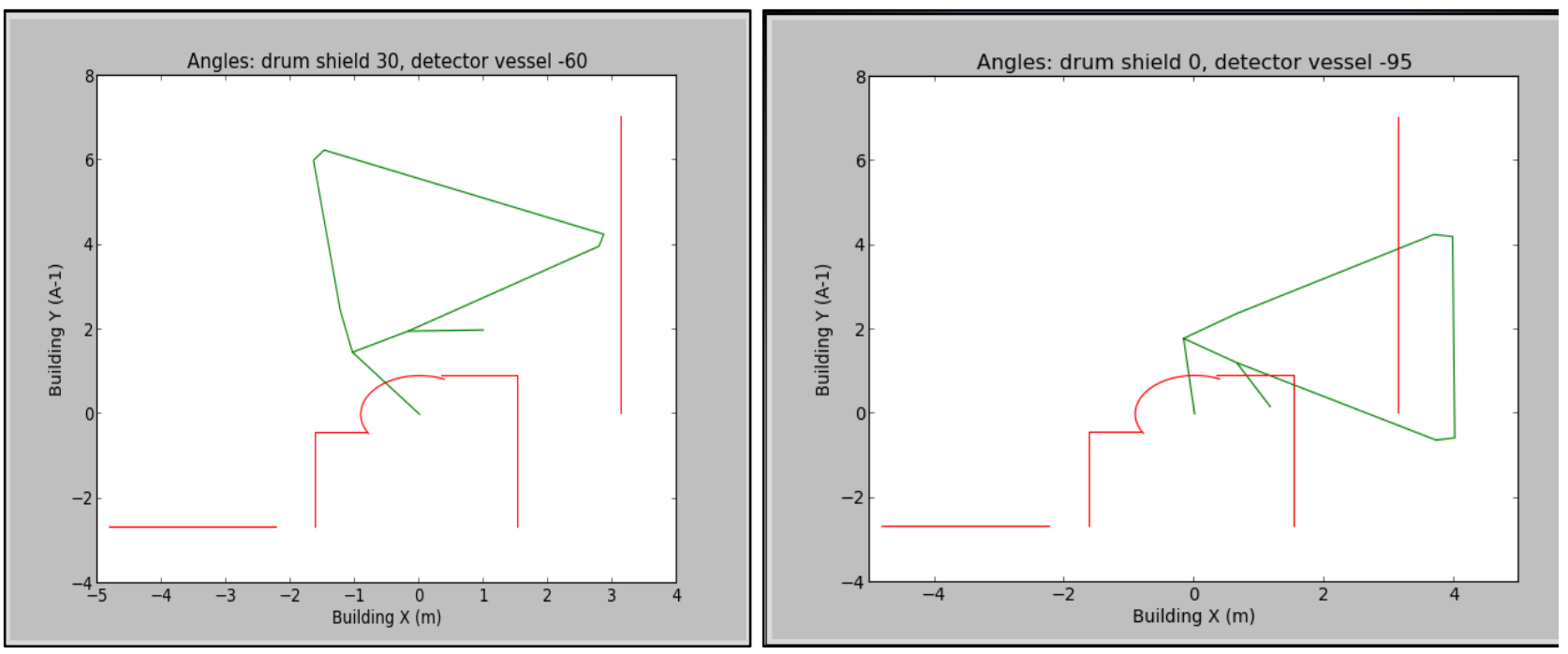

Figure 17: Example of reasonable detector vessel data.

Figure 18: Example of unreasonable detector vessel data. 
excluded where the log data reflected a position that was impossible to physically achieve [Figure 18]. All other aspects of these excluded data sets appeared useable nevertheless the detector vessel positions were an important part of the analysis so any runs that presented $\log$ data that was physically unattainable was excluded. 


\section{APPENDIX C. ELASTIC PEAK FILTER}

HYSPEC has historically endeavored to conduct experiments where the user's TOF data doesn't coincide with the TOF windows occupied by the prompt pulse as the counts recorded during that time would adulterate the desired user data. The efforts of this investigation did nearly the inverse of this. This analysis required data sets that did not contain prompt pulses that were overlapped by elastic or inelastic user data acquired during a particular run. If this were to happen, false intensities could be recorded for the prompt pulse and false correlations could be drawn from the resulting data. To address this, a simple algorithm was implemented to distinguish when and if the prompt pulse was encroached on by the user data.

The maximum value of any given data set will be created by the fraction of incident neutrons scattering elastically from the sample into the detector (the un-scattered fraction is removed by a beamstop). This peak and all satellite peaks were consistently found to occupy no more than approximately $6.0 \mathrm{~ms}$ of data in the $16.667 \mathrm{~ms}$ frame of data collection. Common practice for HYSPEC is to center the $16.667 \mathrm{~ms}$ window about the time of the expected elastic peak [Figure 19] with $\sim 3.0 \mathrm{~ms}$ being before and after the elastic peak to maximize the user's available options for inelastic measurements. With this knowledge you can safely say that if the elastic maximum (maximum of the entire data set) fell within $3.0 \mathrm{~ms}$ of the anticipated $3.0 \mathrm{~ms}$ window for the prompt pulse, that the data for the prompt pulse most likely would have been adulterated by the elastic peak. This was a crude but efficient qualification as the width of these (in)elastic pulses varies for each experiment but was typically found not to occupy more than 6.0ms. Perhaps some data sets were disqualified that didn't truly have adulterated data, but in the interest of efficiency this proved to be an effective way to further refine the data and left the investigation with approximately $70 \%$ of the total runs (approximately 12,500 runs filtered).

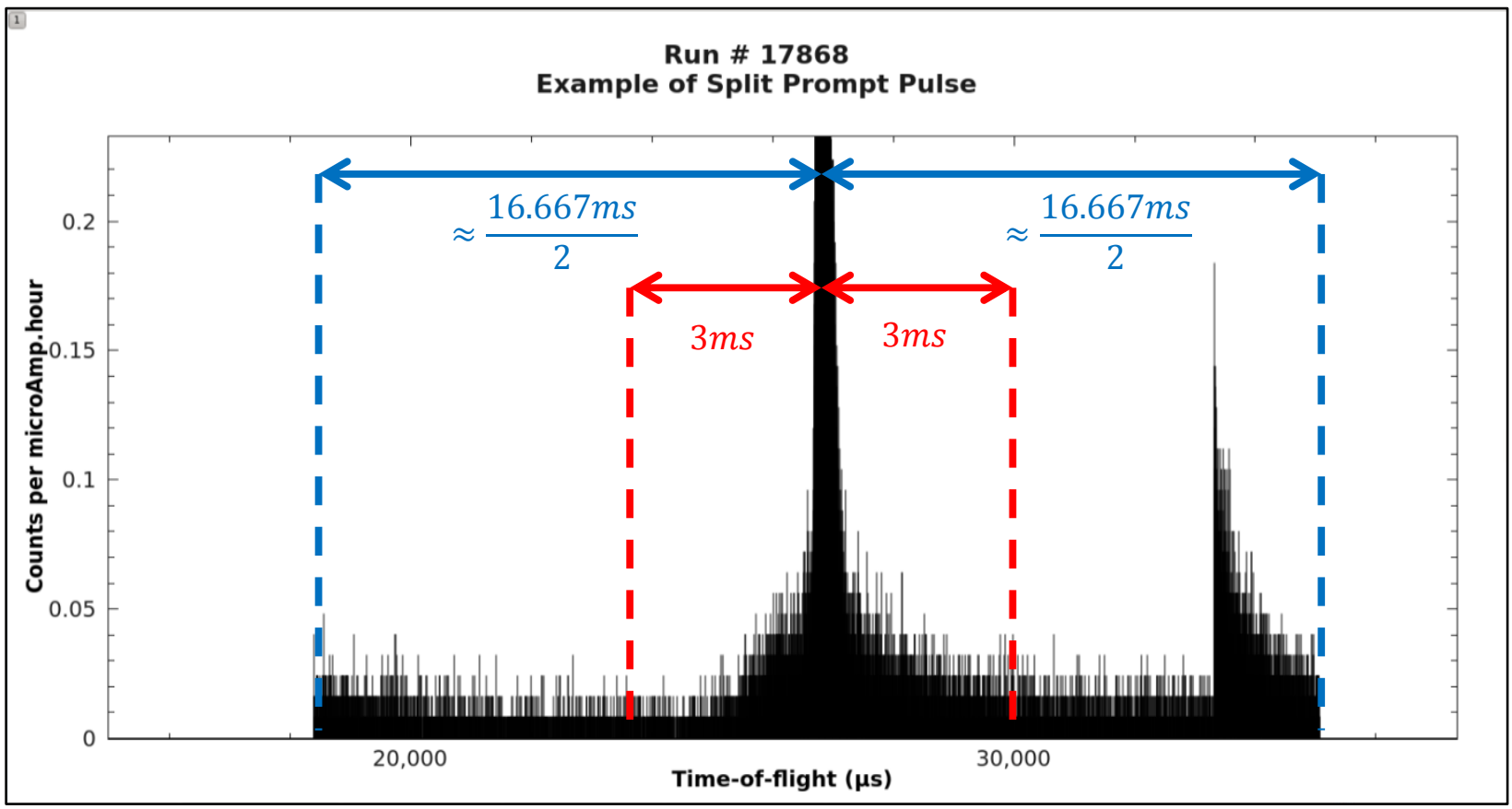

Figure 19: Maximum TOF window expected from elastic data compared to total TOF window for data acquisition. 



\section{APPENDIX D. GAMMA AND INELASTIC PEAK FILTER}

Although the elastic filter was effective in large scale data separation and refinement, it was found that other smaller perturbations were found to adulterate the prompt pulse. During an experiment, it's not unheard of to experience a "gamma flash" from the sample. As the neutrons interact with the sample a portion of them can excite the material to a point to create gamma production and thus send a wave (at the speed of light) from the sample in all directions. A portion of this wave can be detected by HYSPEC's detectors and at times this peak was found to fall outside the $6.0 \mathrm{~ms}$ window of the elastic/inelastic peaks [Figure 20]. Also, extreme cases of inelastic scattering could fall outside the typical $6 \mathrm{~ms}$ window of expected elastic/inelastic data.

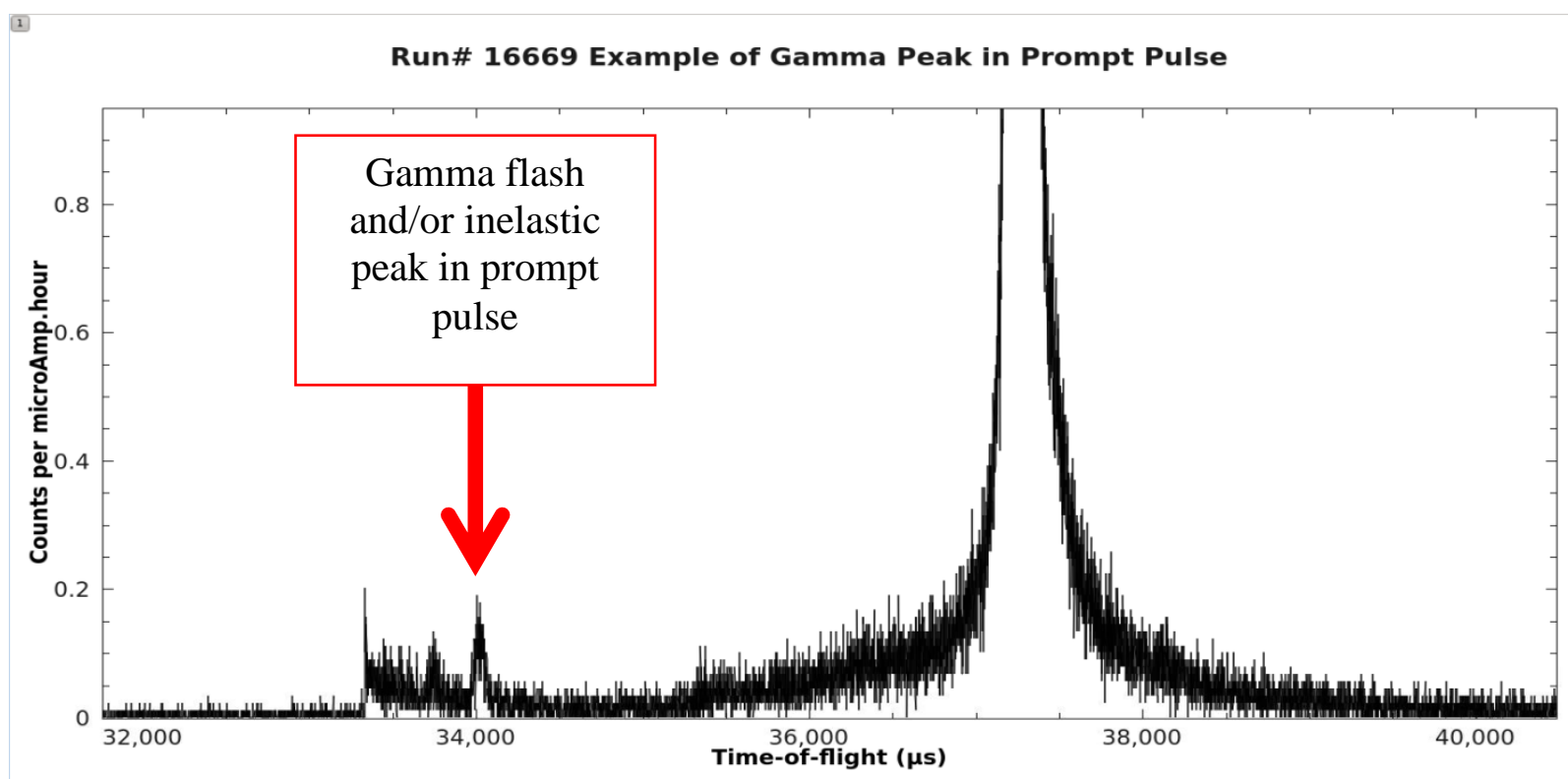

Figure 20: Gamma peak in prompt pulse.

To address these erratic events, the first approach was to simply widen the elastic filter window to accommodate any runs that may include this peak but it was found that although this phenomenon was common it wasn't common enough to warrant the large losses of useable data that would have been excluded by the arbitrary widening of the elastic filter window in anticipation of a peak that only arrived a small percentage of the time. This feature was only noticed after the initial analysis had begun and the filter was applied to further refine the collection of "qualified" runs. This is worth mentioning because the implementation of this filter was made possible by data that had already been acquired. During the analysis the entire prompt pulse was generally monitored by an integration of the $3.0 \mathrm{~ms}$ window of the prompt pulse and that number was used as a metric to observe fluctuation or variations in the overall prompt pulse magnitude. Additionally, the prompt pulse was analyzed on smaller time increments (ie:500 $\mu \mathrm{s}$ bins) in the event that this could prove useful. It turned out to be just that. A quick filter to determine if the prompt pulse was adulterated by the gamma flash was to take these incremental smaller windows of integration and perform a progressive ratio of them. If any of the ratios 
exceeded or are equal to 1.0 (implying an abnormal spike in the tail of the prompt pulse) the run was disqualified [Table 7].

Table 7: Quantitative example of gamma and inelastic filter

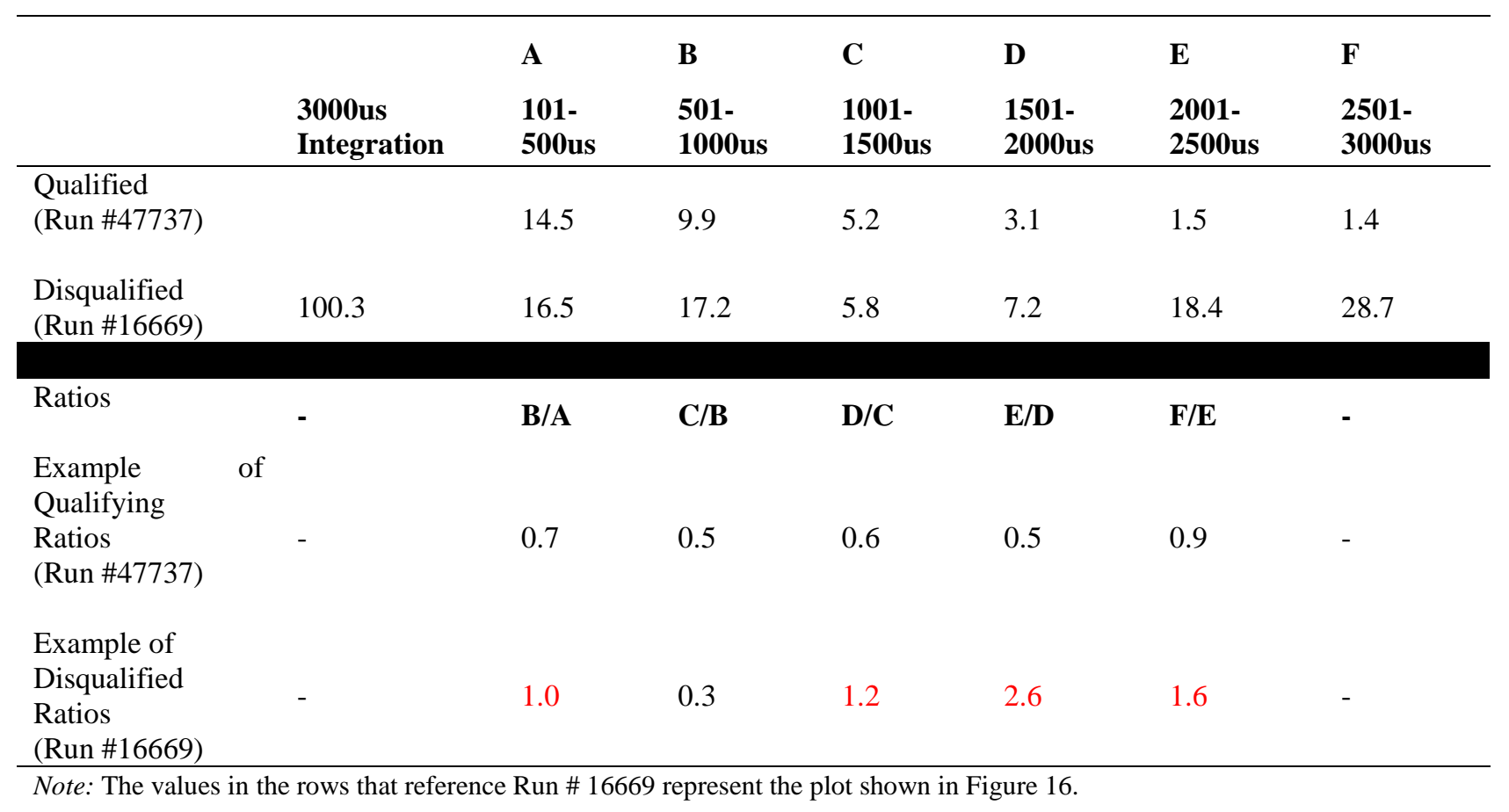

The values in red have been highlighted to show where the subsequent portion of the pulse was larger than the portion that preceded it. It should be mentioned that the particular run displayed in Table 7 has two disqualifications as it does contain a gamma peak (indicated by the red value in the B/A column) but it also shows that it would have been disqualified by the elastic filter as well (indicated by the $\mathrm{D} / \mathrm{C}-\mathrm{F} / \mathrm{E}$ values in red). This is only mentioned as certain filters work in congress with others by serving as a redundancy if one were to perhaps algorithmically overlook an aspect of an unusual data set. This filter was responsible for filtering approximately 5,000 runs from the total amount of available user files (approximately $8 \%$ of the total runs). 


\section{APPENDIX E. SPLIT PULSE RECONSTRUCTION ALGORITHM}

To account for runs where the prompt pulse is split, a portion of the program was modified to first find the prompt pulse in the expected TOF window, then determine what TOF bin the peak (or maximum) of the pulse occupied. Then a sequential counter evaluated each TOF bin from the largest (or longest time) to the smallest (or shortest time). Potential TOF bins beyond the window of acquisition would contain no data so the counter would continue to count down through the bins until it reached the first bin containing data.

Once a bin containing values greater than zero presented its self, it could be declared the last TOF bin that contained data for that run. Since the integration window for the prompt pulse was $3000 \mu \mathrm{s}$, if the difference between the TOF bin of the maximum of the prompt pulse and the last TOF bin in the data collection was less than $3000 \mu \mathrm{s}$, the prompt pulse was split and needed to be reconstructed. This was accomplished by creating an array that sequentially appended the histogram's values per bin (in this case, per $\mu$ s) as the pulse was evaluated in ascending order. If the pulse was split this algorithm would start to append the

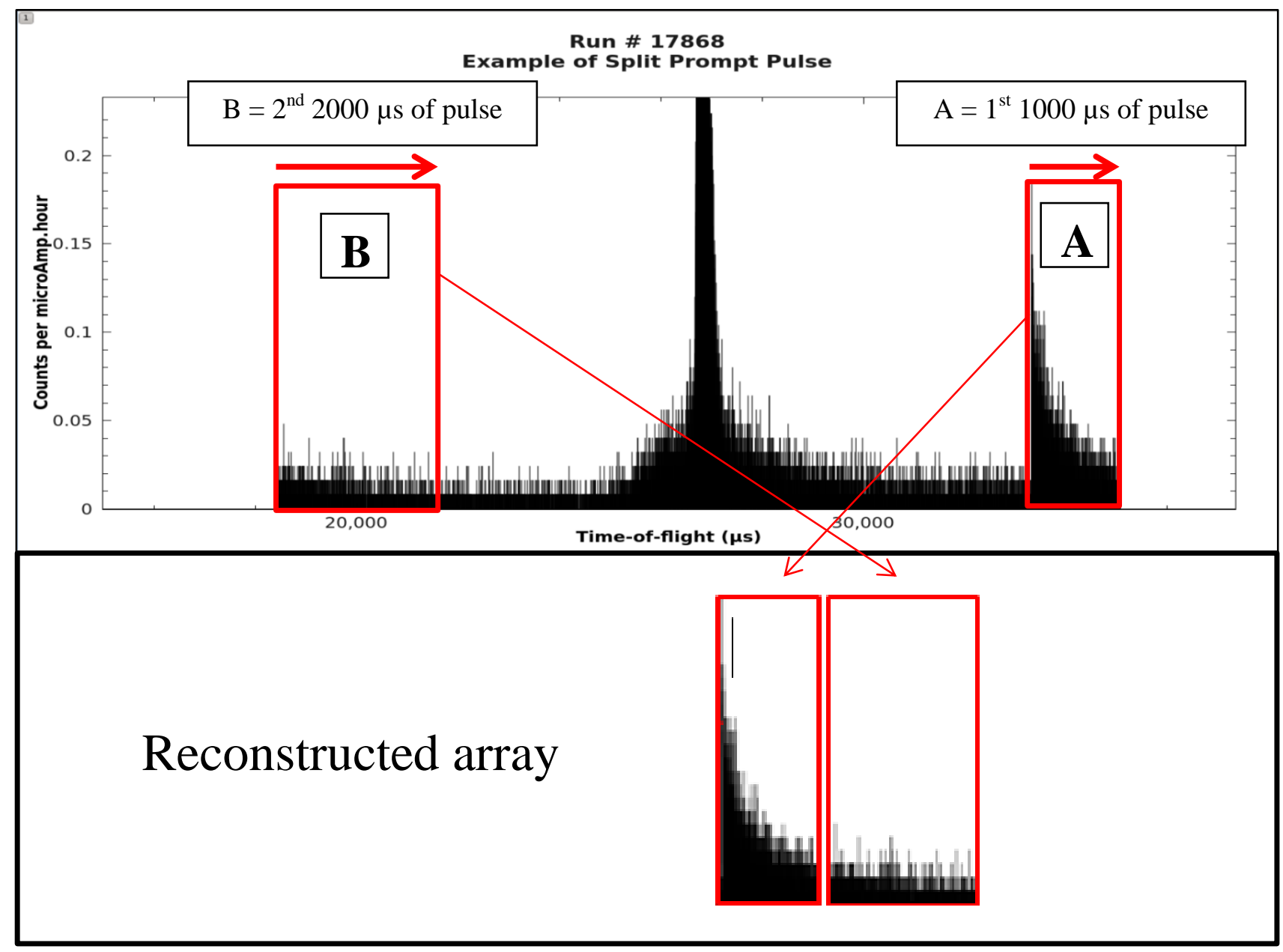

Figure 21: Reconstruction of split prompt pulse. 
beginning of the data acquisition to the end of the previous array thus making a seamless reconstruction of the total prompt pulse [Figure 21]. It should be noted that these split pulses actually represent neutrons from two separate proton impacts and are being incorporated to represent a pulse generating from a single impact. This approach was still decided to be accurate as the data used for these integrations were normalized by charge per pulse (counts $/ \mu \mathrm{A} \cdot \mathrm{hr}$ ) prior to these manipulations. This method was implemented and confirmed to perform the integration accurately and consistently. 


\section{APPENDIX F. DETECTOR CONFIGURATION ALGORITHM}

Appendix B explained the filtering process based on instrument configuration but the actual method of calculating and classifying the instrument's configuration is discussed in this appendix.

Each HYSPEC run is accompanied by a log including various instrument settings, accelerator settings, date and time of data acquisition and much more. These logs were used in determining the region and orientation of the detector and also used to filter out runs where this data was incorrect. As mentioned previously, HYSPEC has the ability to pivot around the room about two axes. These axes are named $\mathrm{s} 2$ for the pivot point about the sample and $\mathrm{m} 2$ for the pivot about the monochromating crystal array [Refer back to Figure 5]. Because the positioning of the detector array plays a role in the changing magnitude of the prompt pulse, an algorithm needed to be developed to classify these parameters. This was accomplished by knowing the physical dimensions of the detector vessel's various components and by obtaining the $\mathrm{m} 2$ and $\mathrm{s} 2$ data for each individual run. The center of the detector array was used as a point to follow about the room and is indicated in Figure 18 by the name "Head $(\mathrm{x}, \mathrm{y})$ ". The room was then broken into four quadrants based on a simple Cartesian coordinate system and the point on the detector was assigned a position ( $\mathrm{x}, \mathrm{y})$ based on its location in the coordinate system. Where these coordinates landed in reference to the quadrants is what classified the detector to be in one "region" or another.

The "orientation" of the vessel was important to define as the "region" alone was not enough to fully isolate all positional variables that HYSPEC could physically accomplish. Orientation was based off a vector relationship with a dynamic part of the instrument compared with a stationary axis of the coordinate system. Since the point in the center of the detector array had already been determined for the classification of the region, that point could now also serve as the $(\mathrm{x}, \mathrm{y})$ coordinate of the head of the dynamic vector. Using a similar method as above, the point that represented the pivot point s2 was also calculated and served as the tail of this "dynamic vector" [Figure 22]. With these vectors defined an angle could be determined relative to this "reference vector" as another means to classify the position(s) of HYSPEC [Figure 22]. Then, parameters were created to classify the various potential orientation angles in the room. Six bins of angle ranges were created and the angle of each individual run was calculated and then placed into the corresponding bin, which defined the "orientation" of the detector vessel at the time of data acquisition [Table 8].

Table 8: Orientation angle ranges for HYSPEC detector vessel

\begin{tabular}{ll}
\hline Orientation \# & Detector Orientation Angle Ranges \\
\hline 1 & $0.0^{0}-22.5^{0}$ \\
2 & $22.5^{0}-45.0^{0}$ \\
3 & $45.1^{0}-67.5^{0}$ \\
4 & $67.6^{0}-112.5^{0}$ \\
5 & $112.6^{0}-157.5^{0}$ \\
6 & $157.6^{0}-202.5^{0}$ \\
\hline
\end{tabular}


Because of the size of HYSPEC and the building enclosing it, there are physical constraints to the possible combinations of "region" and "orientation" that the instrument can actually occupy. Because of this a simple program was written that plotted all available runs by "region" and "orientation" and it was found that out of these two parameters there were only eight possible combinations physically possible. This new term, "configuration", was coined and used to further classify both where and how the detector vessel was positioned inside the room.

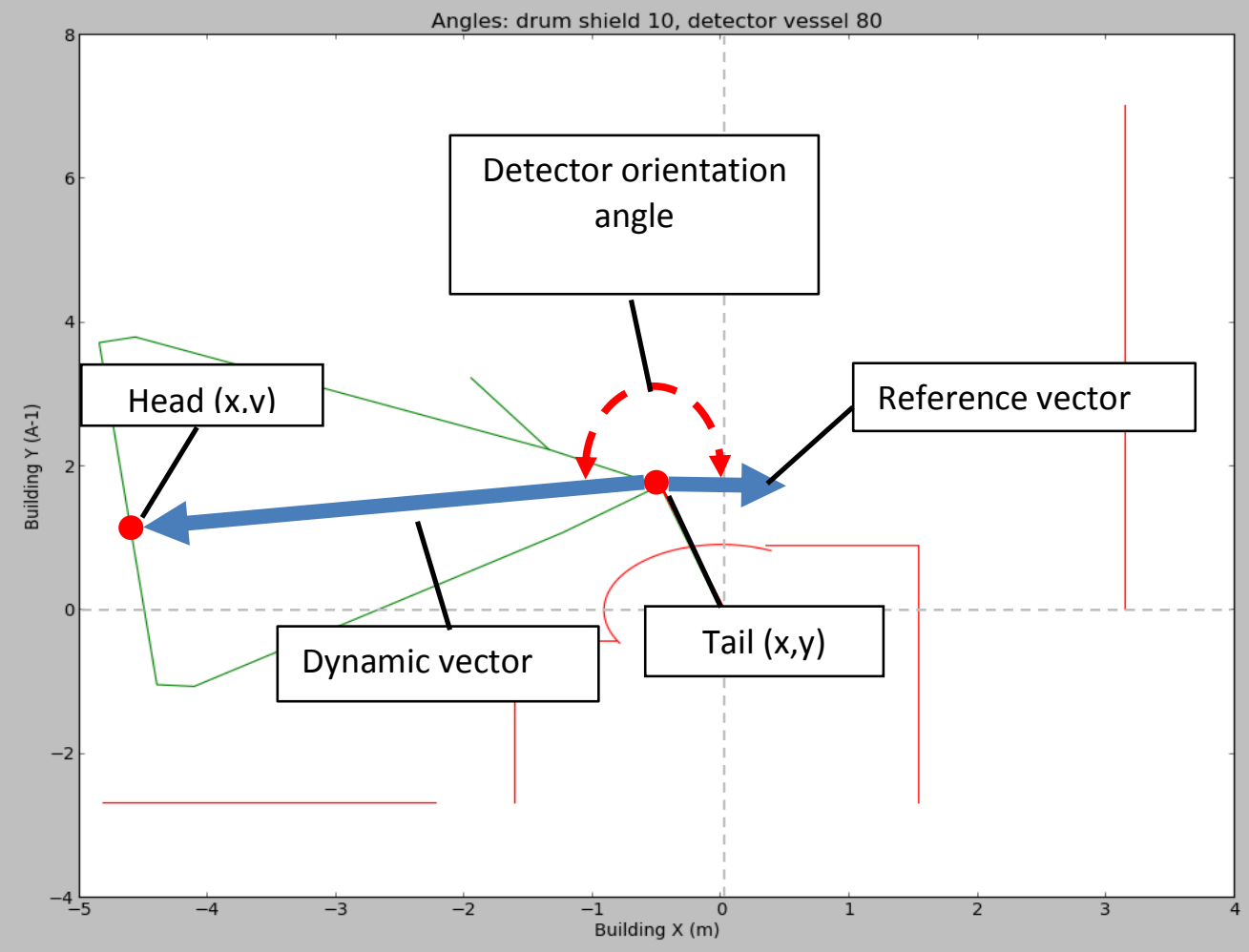

Figure 22: Vector names with "head" and "tail" coordinate locations for orientation. 


\section{APPENDIX G. FREQUENCY DISTRIBUTION ALGORITHM}

The frequency distributions were created from two separate data tables that had previously been produced by our scripts. One table was a collection of values regarding the integration of the prompt pulse along with the run number, date, etc. The other table was a collection of data from the log information; $\mathrm{m} 2$ and s2 angles, configuration, cumulative charge for the run, etc. Each table was accessed and scanned from top to bottom simply determining which configuration was attributed to each $3 \mathrm{~ms}$ integration value. The integration values were placed into an array for each specific configuration and the values were then rounded to the nearest integer. These values were then subjected to a frequency distribution so each configuration's prompt magnitude distribution could be qualitatively observed and statistically evaluated. This process expedited the process of identifying configurations that were affected and/or unaffected by outside influences and also helped to solidify the influence of HYSPEC's configuration. 



\section{APPENDIX H. ACCESSING AND IMPORTING CSS DATA INTO MANTIDPLOT}

CSS was accessed and specific process variables (PV's) were investigated within the CSS software. Shutter data was typically being investigated in this analysis and the data was convenient in a sense that the values representing date and time-stamped shutter position data are either a 1 (shutter open) or a 0 (shutter closed). This was helpful in that our investigation required data from 2011 (the inception of HYSPEC) to the present day ( 2014). This required a long series of ones, zeroes and corresponding date/times that could be exported from CSS into a comma separated value (CSV) file and then ultimately read in by the Python scripts to Mantidplot. This data was now in a format where a comparison of the time of data acquisition and time of shutter position could be made.

More data was lost during this comparison/qualification as our analysis required HYSPEC runs where the shutter was either completely shut or completely closed during the run, not in transition. Once this task was performed, a comprehensive list could be made where all known variables (all shutters and HYSPEC configuration) could be held constant. 



\section{APPENDIX I. CALCULATIONS AND STATISTICS FORMULAE FOR SPECIFIC BEAMLINE} AND INTERNAL CONFIGURATION CONTRIBUTIONS

The following tables show the actual data used to calculate the values shown in Table $4 \& 5$. The values in the following tables were calculated as follows:

1. The number of runs for All-Open $\left(N_{O}\right)$ and Only-One-Closed $\left(N_{X X}\right)$ : This is how many runs were used for a given calculation in a particular configuration for all shutters open $\left(N_{O}\right)$ and when only one was closed $\left(N_{X X}\right)$.

2. The magnitude of a single prompt pulse for All-Open $\left(P_{O(\#)}\right)$ and Only-One-Closed $\left(P_{X X(\#)}\right):$ This value represents the $3 \mathrm{~ms}$ integration of the counts/ $\mu \mathrm{A} \cdot \mathrm{hr}$ for a single run. The data was binned by $1 \mu$ s bins.

3. All-Open mean $\left(\mu_{O(\#)}\right)$ and Only-One-Closed mean $\left(\mu_{X X(\#)}\right)$ : This value represents the mean of the prompt pulse magnitude when all the shutters in this analysis were open/closed. It was calculated by the standard form of a mean where:

$$
\mu_{O(\#)}=\frac{\sum\left(P_{O(\#)}\right)}{N_{O}} \quad \mu_{X X(\#)}=\frac{\sum\left(P_{X X(\#)}\right)}{N_{X X}}
$$

4. Width of the distribution, All-Open $\left(\sigma_{\mathrm{O}(\#)}\right)$ and Only-One-Closed $\left(\sigma_{\mathrm{XX}(\#)}\right)$ : This value was used to observe the standard deviation of the distributions and was calculated using:

$$
\sigma_{\mathrm{O}(\#)}=\sqrt{\frac{\sum\left(P_{O(\#)}-\mu_{O(\#)}\right)^{2}}{N_{O}-1}} \quad \sigma_{\mathrm{XX}(\#)}=\sqrt{\frac{\sum\left(P_{X X(\#)}-\mu_{X X(\#)}\right)^{2}}{N_{X X}-1}}
$$

5. Standard Deviation of the Mean, All-Open $\left(\Omega_{\mathrm{O}(\#)}\right)$ and Only-One-Closed $\left(\Omega_{\mathrm{XX}(\#)}\right)$ : This value was used to observe the standard deviation of the mean of the distribution and was calculated using:

$$
\Omega_{\mathrm{O}(\#)}=\frac{\sigma_{\mathrm{O}(\#)}}{\sqrt{N_{O}}} \quad \Omega_{\mathrm{XX}(\#)}=\frac{\sigma_{\mathrm{XX}(\#)}}{\sqrt{N_{X X}}}
$$

6. The difference of the means $\left(C_{X X(\#)}\right)$ : These values are simply each difference between the mean of the "Only One Closed (Mean)" and the "All Open (Mean)".

$$
C_{X X(\#)}=\mu_{O(\#)}-\mu_{X X(\#)}
$$


7. Width of the difference of the means $\left(\sigma_{\mathrm{CXX}(\#)}\right)$ : This was the combined standard deviation of the distribution and was found by summing the two respective distribution's deviations in quadrature:

$$
\sigma_{\mathrm{CXX}(\#)}=\sqrt{\sigma_{O(\#)}^{2}+\sigma_{X X(\#)}^{2}}
$$

8. Total uncertainty in the difference of the means $\left(\Omega_{\operatorname{CXX}(\#)}\right)$ : This was the combined standard deviation of the means of the distributions and was found by summing the two respective mean's deviations in quadrature:

$$
\Omega_{\mathrm{CXX}(\#)}=\sqrt{\Omega_{O(\#)}^{2}+\Omega_{X X(\#)}^{2}}
$$

The following tables show the individual data for each respective beamline's shutter position and HYSPEC's instrument configuration. Also, the CSS process variable (PV's) names corresponding to all beamlines are listed within each beamline's table. It should be noted that some beamlines utilize a single "primary" shutter while others use a "primary" and a secondary. The beamlines with two shutters will have a table showing the impact of each shutter independently, while beamline's consisting of a single "primary" shutter will only have one table.

Table 9: Calculations for prompt pulse magnitude when all north side shutters are open

\begin{tabular}{llllll}
\hline Configuration & \multicolumn{1}{c}{$\boldsymbol{\mu}_{\boldsymbol{O}(\#)}$} & & $\boldsymbol{\sigma}_{\mathbf{O}(\#)}$ & $\boldsymbol{\Omega}_{\mathbf{O}(\#)}$ & $\boldsymbol{N}_{\mathbf{0}}$ \\
\hline 1 & 43.3 & 2.7 & 0.1 & 489 \\
2 & - & - & - & - \\
3 & 40.8 & 3.2 & 0.1 & 2104 \\
4 & 41.0 & 1.9 & 0.5 & 15 \\
5 & 38.7 & 1.4 & 0.1 & 107 \\
6 & 36.1 & 2.4 & 0.1 & 1996 \\
7 & 35.4 & 3.0 & 2.1 & 2 \\
8 & 32.8 & 4.7 & 0.3 & 250 \\
\hline
\end{tabular}


Table 10: Calculations for prompt pulse magnitude where BL-11 primary shutter is the only one closed

Beamline 11 Primary Shutter Only One Closed

CSS PV: Tgt_shld:Shtr7711:SwOpen

\begin{tabular}{|c|c|c|c|c|c|c|c|}
\hline Configuration & $\mu_{11 a(\#)}$ & $\sigma_{11 \mathrm{a}(\#)}$ & $\mathbf{\Omega}_{11 \mathrm{a}(\#)}$ & $C_{11 a(\#)}$ & $\sigma_{\mathrm{C} \mathrm{11a}(\#)}$ & $\mathbf{\Omega}_{\mathrm{C} \text { 11a(\#) }}$ & $N_{11 a(\#)}$ \\
\hline 1 & - & - & - & - & - & - & - \\
\hline 2 & - & - & - & - & - & - & - \\
\hline 3 & - & - & - & - & - & - & - \\
\hline 4 & - & - & - & - & - & - & - \\
\hline 5 & - & - & - & - & - & - & - \\
\hline 6 & - & - & - & - & - & - & - \\
\hline 7 & - & - & - & - & - & - & - \\
\hline 8 & - & - & - & - & - & - & - \\
\hline
\end{tabular}

Table 11: Calculations for prompt pulse magnitude where BL-11a secondary shutter is the only one closed

\begin{tabular}{|c|c|c|c|c|c|c|c|}
\hline \multicolumn{8}{|c|}{$\begin{array}{l}\text { Beamline 11a Secondary Shutter Only One Closed } \\
\text { CSS PV: PPS_BMLN:SHSC11a_01:OPN }\end{array}$} \\
\hline Configuration & $\mu_{11 a(\#)}$ & $\sigma_{11 \mathrm{a}(\#)}$ & $\mathbf{\Omega}_{11 \mathrm{a}(\#)}$ & $C_{11 a(\#)}$ & $\sigma_{\mathrm{C} 11 \mathrm{a}(\#)}$ & $\Omega_{\mathrm{C} 11 \mathrm{a}(\#)}$ & $N_{11 a(\#)}$ \\
\hline 1 & 43.6 & 2.1 & 0.5 & -0.2 & 3.4 & 0.5 & 16 \\
\hline 2 & - & - & - & - & - & - & - \\
\hline 3 & 40.7 & 1.9 & 0.2 & 0.1 & 3.7 & 0.2 & 66 \\
\hline 4 & 34.2 & 0.0 & 0.0 & 6.8 & 1.9 & 0.5 & 1 \\
\hline 5 & 38.2 & 0.6 & 0.4 & 0.5 & 1.6 & 0.4 & 3 \\
\hline 6 & 36.6 & 1.7 & 0.5 & -0.4 & 2.9 & 0.5 & 13 \\
\hline 7 & - & - & - & - & - & - & - \\
\hline 8 & - & - & - & - & - & - & - \\
\hline
\end{tabular}

Table 12: Calculations for prompt pulse magnitude where BL-11b secondary shutter is the only one closed

\begin{tabular}{|c|c|c|c|c|c|c|c|}
\hline \multicolumn{8}{|c|}{$\begin{array}{l}\text { Beamline 11b Secondary Shutter Only One Closed } \\
\text { CSS PV: PPS_BMLN:SHSC11b_01:OPN }\end{array}$} \\
\hline Configuration & $\mu_{11 b(\#)}$ & $\sigma_{11 b(\#)}$ & $\boldsymbol{\Omega}_{11 \mathrm{~b}(\#)}$ & $C_{11 b(\#)}$ & $\sigma_{\mathrm{C} 11 \mathbf{b}(\#)}$ & $\Omega_{\mathrm{C} 11 \mathrm{~b}(\#)}$ & $N_{11 b(\#)}$ \\
\hline 1 & - & - & - & - & - & - & - \\
\hline 2 & - & - & - & - & - & - & - \\
\hline 3 & 39.7 & 2.0 & 0.1 & 1.1 & 3.8 & 0.1 & 1280 \\
\hline 4 & - & - & - & - & - & - & - \\
\hline 5 & - & - & - & - & - & - & - \\
\hline 6 & 34.7 & 2.2 & 0.1 & 1.5 & 3.2 & 0.1 & 529 \\
\hline 7 & - & - & - & - & - & - & - \\
\hline 8 & - & - & - & - & - & - & - \\
\hline
\end{tabular}


Table 13: Calculations for prompt pulse magnitude where BL-12 primary shutter is the only one closed

\begin{tabular}{|c|c|c|c|c|c|c|c|}
\hline \multicolumn{8}{|c|}{$\begin{array}{l}\text { Beamline } 12 \text { Primary Shutter Only One Closed } \\
\text { CSS PV: Tgt_shld:Shtr7712:SwOpen }\end{array}$} \\
\hline Configuration & $\boldsymbol{\mu}_{12(\#)}$ & $\sigma_{12(\#)}$ & $\mathbf{\Omega}_{\mathbf{1 2}(\#)}$ & $C_{12(\#)}$ & $\sigma_{\mathrm{C} 12(\#)}$ & $\Omega_{\mathrm{C} 12(\#)}$ & $N_{12(\#)}$ \\
\hline 1 & - & - & - & - & - & - & - \\
\hline 2 & - & - & - & - & - & - & - \\
\hline 3 & 34.6 & 2.2 & 0.1 & 6.3 & 3.9 & 0.1 & 457 \\
\hline 4 & - & - & - & - & - & - & - \\
\hline 5 & - & - & - & - & - & - & - \\
\hline 6 & 36.1 & 2.9 & 0.3 & 0.0 & 3.8 & 0.3 & 75 \\
\hline 7 & - & - & - & - & - & - & - \\
\hline 8 & 30.8 & 1.4 & 0.1 & 2.0 & 4.9 & 0.3 & 148 \\
\hline
\end{tabular}

Table 14: Calculations for prompt pulse magnitude where BL-13 primary shutter is the only one closed

\begin{tabular}{|c|c|c|c|c|c|c|c|}
\hline \multicolumn{8}{|c|}{$\begin{array}{l}\text { Beamline } 13 \text { Primary Shutter Only One Closed } \\
\text { CSS PV: Tgt_shld:Shtr7713:SwOpen }\end{array}$} \\
\hline Configuration & $\mu_{13(\#)}$ & $\sigma_{13(\#)}$ & $\mathbf{\Omega}_{\mathbf{1 3}(\#)}$ & $C_{13(\#)}$ & $\sigma_{\mathrm{C} 13(\#)}$ & $\mathbf{\Omega}_{\mathrm{C} 13(\#)}$ & $N_{13(\#)}$ \\
\hline 1 & 30.0 & 1.1 & 0.1 & 13.3 & 2.7 & 0.1 & 320 \\
\hline 2 & - & - & - & - & - & - & - \\
\hline 3 & 31.3 & 1.4 & 0.1 & 9.6 & 7.2 & 0.1 & 659 \\
\hline 4 & - & - & - & - & - & - & - \\
\hline 5 & 31.2 & 1.1 & 0.1 & 7.4 & 2.5 & 0.2 & 165 \\
\hline 6 & 29.8 & 1.4 & 0.1 & 6.3 & 3.9 & 0.1 & 320 \\
\hline 7 & 28.2 & 1.2 & 0.1 & 7.2 & 3.5 & 2.1 & 108 \\
\hline 8 & - & - & - & - & - & - & - \\
\hline
\end{tabular}

Table 15: Calculations for prompt pulse magnitude where BL-13 secondary shutter is the only one closed

\begin{tabular}{|c|c|c|c|c|c|c|c|}
\hline \multicolumn{8}{|c|}{$\begin{array}{l}\text { Beamline } 13 \text { Secondary Shutter Only One Closed } \\
\text { CSS PV: PPS_BMLN:SHSC13_01:OPN }\end{array}$} \\
\hline Configuration & $\mu_{13(\#)}$ & $\sigma_{13(\#)}$ & $\mathbf{\Omega}_{\mathbf{1 3}(\#)}$ & $C_{13(\#)}$ & $\sigma_{\mathrm{C} 13(\#)}$ & $\Omega_{\mathrm{C} 13(\#)}$ & $N_{13(\#)}$ \\
\hline 1 & 32.5 & 0.2 & 0.1 & 10.8 & 2.7 & 0.1 & 9 \\
\hline 2 & - & - & - & - & - & - & - \\
\hline 3 & 31.9 & 6.5 & 0.3 & 8.9 & 7.2 & 0.3 & 542 \\
\hline 4 & - & - & - & - & - & - & - \\
\hline 5 & 31.4 & 2.1 & 0.5 & 7.3 & 2.5 & 0.5 & 17 \\
\hline 6 & 28.3 & 3.1 & 0.4 & 7.8 & 3.9 & 0.4 & 51 \\
\hline 7 & 29.6 & 1.7 & 0.8 & 5.8 & 3.5 & 2.3 & 5 \\
\hline 8 & - & - & - & - & - & - & - \\
\hline
\end{tabular}


Table 16: Calculations for prompt pulse magnitude where BL-15 primary shutter is the only one closed

\begin{tabular}{|c|c|c|c|c|c|c|c|}
\hline \multicolumn{8}{|c|}{$\begin{array}{l}\text { Beamline } 15 \text { Primary Shutter Only One Closed } \\
\text { CSS PV: Tgt_shld:Shtr7715:SwOpen }\end{array}$} \\
\hline Configuration & $\boldsymbol{\mu}_{15(\#)}$ & $\sigma_{15(\#)}$ & $\boldsymbol{\Omega}_{\mathbf{1 5}(\#)}$ & $C_{15(\#)}$ & $\sigma_{\mathrm{C} 15(\#)}$ & $\Omega_{\mathrm{C} 15(\#)}$ & $N_{15(\#)}$ \\
\hline 1 & - & - & - & - & - & - & - \\
\hline 2 & - & - & - & - & - & - & - \\
\hline 3 & - & - & - & - & - & - & - \\
\hline 4 & - & - & - & - & - & - & - \\
\hline 5 & - & - & - & - & - & - & - \\
\hline 6 & - & - & - & - & - & - & - \\
\hline 7 & - & - & - & - & - & - & - \\
\hline 8 & - & - & - & - & - & - & - \\
\hline
\end{tabular}

Table 17: Calculations for prompt pulse magnitude where BL-15 secondary shutter is the only one closed

\begin{tabular}{|c|c|c|c|c|c|c|c|}
\hline \multicolumn{8}{|c|}{$\begin{array}{l}\text { Beamline } 15 \text { Secondary Shutter Only One Closed } \\
\text { CSS PV: PPS_BMLN:SHSC15_01:OPN }\end{array}$} \\
\hline Configuration & $\mu_{15(\#)}$ & $\sigma_{15(\#)}$ & $\mathbf{\Omega}_{\mathbf{1 5}(\#)}$ & $C_{15(\#)}$ & $\sigma_{\mathrm{C} 15(\#)}$ & $\Omega_{\mathrm{C} 15(\#)}$ & $N_{15(\#)}$ \\
\hline 1 & 45.2 & 1.9 & 0.4 & -1.8 & 3.3 & 0.4 & 24 \\
\hline 2 & - & - & - & - & - & - & - \\
\hline 3 & 40.1 & 3.4 & 0.2 & 0.7 & 4.7 & 0.2 & 323 \\
\hline 4 & - & - & - & - & - & - & - \\
\hline 5 & 38.2 & 1.0 & 0.3 & 0.4 & 1.7 & 0.3 & 13 \\
\hline 6 & 34.6 & 3.0 & 0.3 & 1.5 & 3.8 & 0.3 & 97 \\
\hline 7 & - & - & - & - & - & - & - \\
\hline 8 & 33.4 & 2.0 & 0.2 & -0.6 & 5.1 & 0.4 & 93 \\
\hline
\end{tabular}

Table 18: Calculations for prompt pulse magnitude where BL-16 primary shutter is the only one closed

\begin{tabular}{|c|c|c|c|c|c|c|c|}
\hline \multicolumn{8}{|c|}{$\begin{array}{l}\text { Beamline 16 Primary Shutter Only One Closed } \\
\text { CSS PV: Tgt_shld:Shtr7716:SwOpen }\end{array}$} \\
\hline Configuration & $\mu_{16(\#)}$ & $\sigma_{16(\#)}$ & $\mathbf{\Omega}_{\mathbf{1 6}(\#)}$ & $C_{16(\#)}$ & $\sigma_{\mathrm{C} 16(\#)}$ & $\Omega_{\mathrm{C} 16(\#)}$ & $N_{16(\#)}$ \\
\hline 1 & - & - & - & - & - & - & - \\
\hline 2 & - & - & - & - & - & - & - \\
\hline 3 & 41.5 & 1.4 & 0.1 & -0.6 & 4.1 & 0.1 & 295 \\
\hline 4 & - & - & - & - & - & - & - \\
\hline 5 & - & - & - & - & - & - & - \\
\hline 6 & 37.5 & 0.0 & 0.0 & -1.4 & 3.2 & 0.1 & 1 \\
\hline 7 & - & - & - & - & - & - & - \\
\hline 8 & 34.0 & 0.2 & 0.1 & -1.2 & 4.8 & 0.3 & 5 \\
\hline
\end{tabular}


Table 19: Calculations for prompt pulse magnitude where BL-16 secondary shutter is the only one closed

\begin{tabular}{|c|c|c|c|c|c|c|c|}
\hline \multicolumn{8}{|c|}{$\begin{array}{l}\text { Beamline 16 Secondary Shutter Only One Closed } \\
\text { CSS PV: PPS_BMLN:SHSC16_01:OPN }\end{array}$} \\
\hline Configuration & $\mu_{16(\#)}$ & $\sigma_{16(\#)}$ & $\mathbf{\Omega}_{16(\#)}$ & $C_{16(\#)}$ & $\sigma_{\mathrm{C} 16(\#)}$ & $\Omega_{\mathrm{C} 16(\#)}$ & $N_{16(\#)}$ \\
\hline 1 & 42.0 & 1.9 & 0.2 & 1.3 & 3.3 & 0.2 & 133 \\
\hline 2 & - & - & - & - & - & - & - \\
\hline 3 & 39.5 & 2.6 & 0.1 & 1.3 & 4.1 & 0.1 & 391 \\
\hline 4 & - & - & - & - & - & - & - \\
\hline 5 & 39.0 & 1.5 & 0.2 & -0.4 & 2.1 & 0.3 & 49 \\
\hline 6 & 36.3 & 2.2 & 0.2 & -0.2 & 3.2 & 0.2 & 150 \\
\hline 7 & 38.9 & 0.0 & 0.0 & -3.5 & 3.0 & 2.1 & 1 \\
\hline 8 & 35.2 & 1.0 & 0.1 & -2.3 & 4.8 & 0.3 & 119 \\
\hline
\end{tabular}

Table 20: Calculations for prompt pulse magnitude where BL-17 primary shutter is the only one closed

\begin{tabular}{|c|c|c|c|c|c|c|c|}
\hline \multicolumn{8}{|c|}{$\begin{array}{l}\text { Beamline } 17 \text { Primary Shutter Only One Closed } \\
\text { CSS PV: Tgt_shld:Shtr7717:SwOpen }\end{array}$} \\
\hline Configuration & $\boldsymbol{\mu}_{17(\#)}$ & $\sigma_{17(\#)}$ & $\mathbf{\Omega}_{\mathbf{1 7}(\#)}$ & $C_{17(\#)}$ & $\sigma_{\mathrm{C} 17(\#)}$ & $\boldsymbol{\Omega}_{\mathrm{C} 17(\#)}$ & $N_{17(\#)}$ \\
\hline 1 & 41.1 & 1.2 & 0.2 & 2.2 & 2.9 & 0.3 & 24 \\
\hline 2 & - & - & - & - & - & - & - \\
\hline 3 & 39.9 & 2.6 & 0.1 & 0.9 & 4.1 & 0.2 & 355 \\
\hline 4 & - & - & - & - & - & - & - \\
\hline 5 & 37.7 & 0.5 & 0.1 & 1.0 & 1.5 & 0.2 & 15 \\
\hline 6 & 35.7 & 2.7 & 0.4 & 0.4 & 3.6 & 0.4 & 52 \\
\hline 7 & - & - & - & - & - & - & - \\
\hline 8 & 33.6 & 0.1 & 0.0 & -0.7 & 4.7 & 0.3 & 2 \\
\hline
\end{tabular}

Table 21: Calculations for prompt pulse magnitude where BL-18 primary shutter is the only one closed

\begin{tabular}{|c|c|c|c|c|c|c|c|}
\hline \multicolumn{8}{|c|}{$\begin{array}{l}\text { Beamline } 18 \text { Primary Shutter Only One Closed } \\
\text { CSS PV: Tgt_shld:Shtr7718:SwOpen }\end{array}$} \\
\hline Configuration & $\mu_{18(\#)}$ & $\sigma_{18(\#)}$ & $\mathbf{\Omega}_{\mathbf{1 8}(\#)}$ & $C_{18(\#)}$ & $\sigma_{\mathrm{C} 18(\#)}$ & $\Omega_{\mathrm{C} 18(\#)}$ & $N_{18(\#)}$ \\
\hline 1 & 48.5 & 12.0 & 2.2 & -5.2 & 12.3 & 2.2 & 30 \\
\hline 2 & - & - & - & - & - & - & - \\
\hline 3 & 40.2 & 4.6 & 0.7 & 0.6 & 5.6 & 0.7 & 39 \\
\hline 4 & - & - & - & - & - & - & - \\
\hline 5 & 38.1 & 0.4 & 0.2 & 0.5 & 1.5 & 0.2 & 6 \\
\hline 6 & 37.4 & 9.0 & 2.3 & -1.3 & 9.3 & 2.3 & 15 \\
\hline 7 & - & - & - & - & - & - & - \\
\hline 8 & 40.3 & 2.5 & 1.7 & -7.4 & 5.3 & 1.8 & 2 \\
\hline
\end{tabular}




\section{APPENDIX J. FREQUENCY DISTRIBUTION GRAPHS}

The following charts show examples of only the most prominent effects of the frequency distribution method for each configuration. For exact numbers of all configuration and shutter combinations refer to Appendix I.

Also, it should be noted that the following images are separated by whether shutters affected the "all-shutters-open" status or not, that is, if a shutter position caused a frequency distribution to move out from under the "All Shutters Open" (black dashed) line than the shutter did have a significant effect on the prompt pulse magnitude. It should also be noted that if a peak shifts to the right on the $\mathrm{x}$-axis that this indicates the prompt pulse magnitude is increasing as a result of the particular shutter being closed. Conversely a leftward shift indicates a decrease in prompt pulse magnitude caused by the shutter being closed. Configurations 2, 4 and 7 are not included in the plots below as they did not provide sufficient statistics for useful graphical representation. Note that the Y-axis scales are not consistent from graph to graph as some configurations had much less statistical representation in these frequency distributions. Despite that, this appendix aims to highlight the differences in "All Shutters Open" VS "Shutter XX Only One Closed" per configuration, not the differences from configuration to configuration.

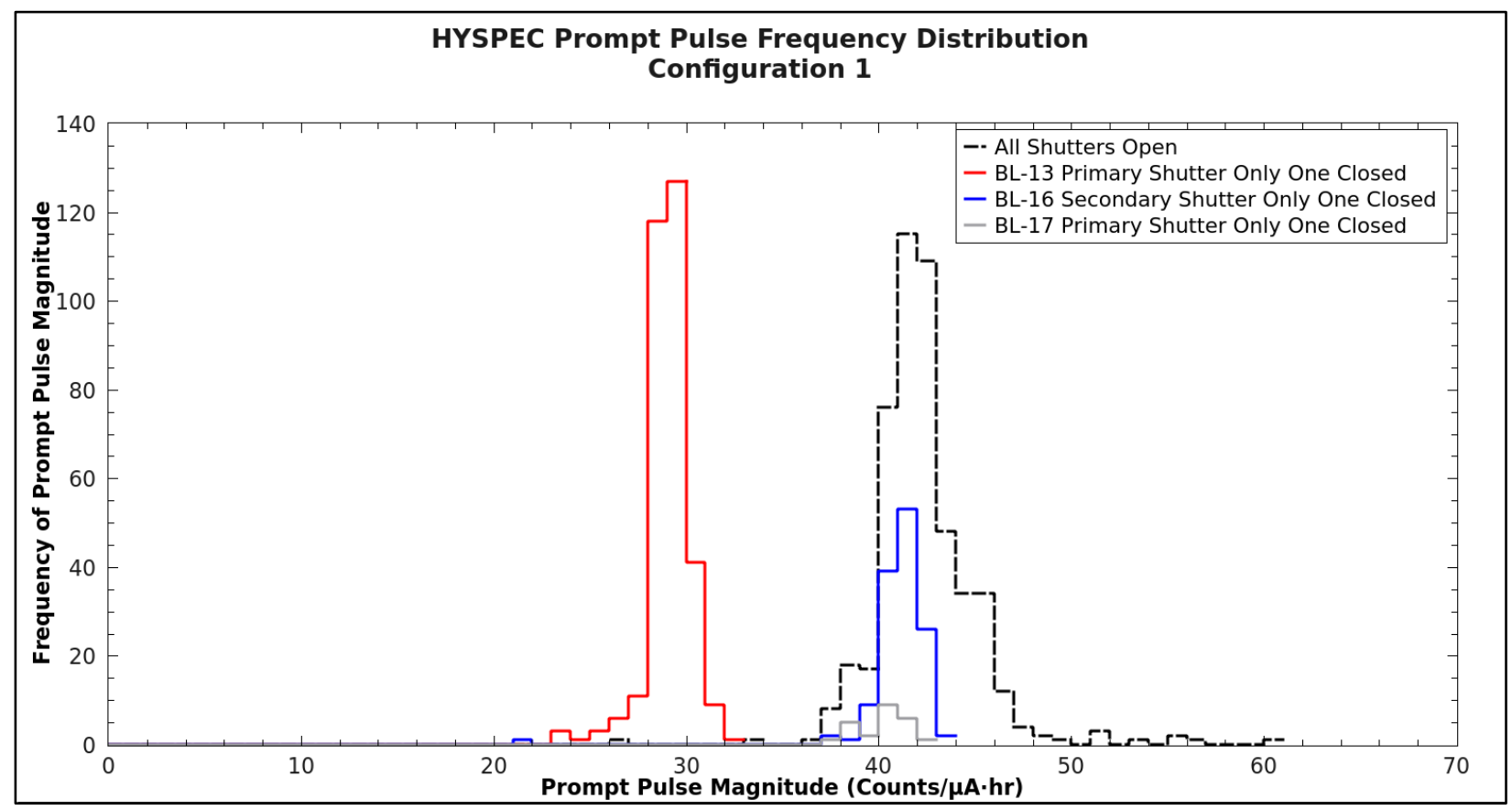

Figure 23: HYSPEC in configuration 1. Comparing background levels when all shutters on the north side of the target facility were open to when certain beamlines were the only ones closed. 


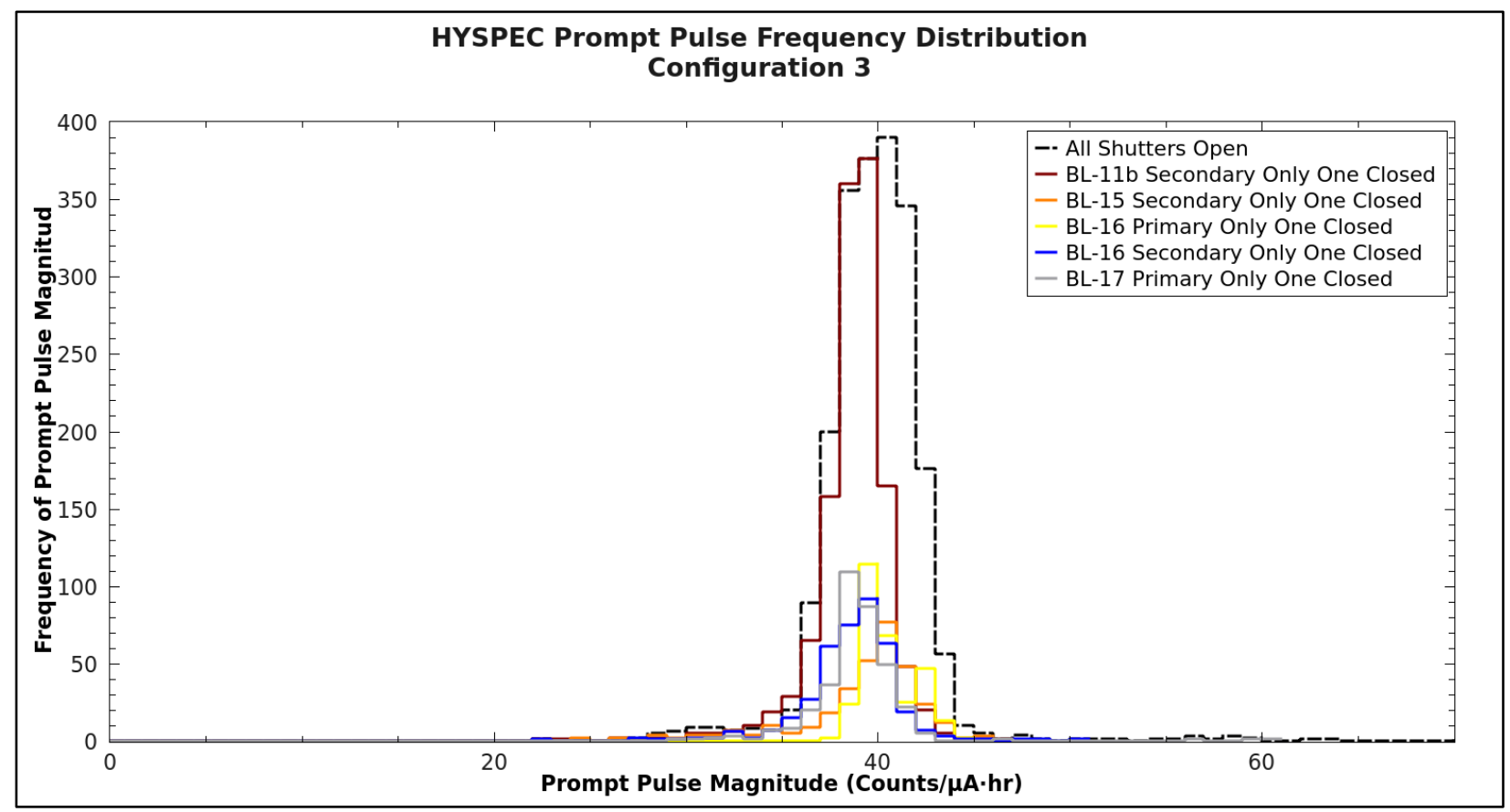

Figure 24: HYSPEC in configuration 3. Comparing background levels when all shutters on the north side of the target facility were open to when certain beamlines were the only ones closed.

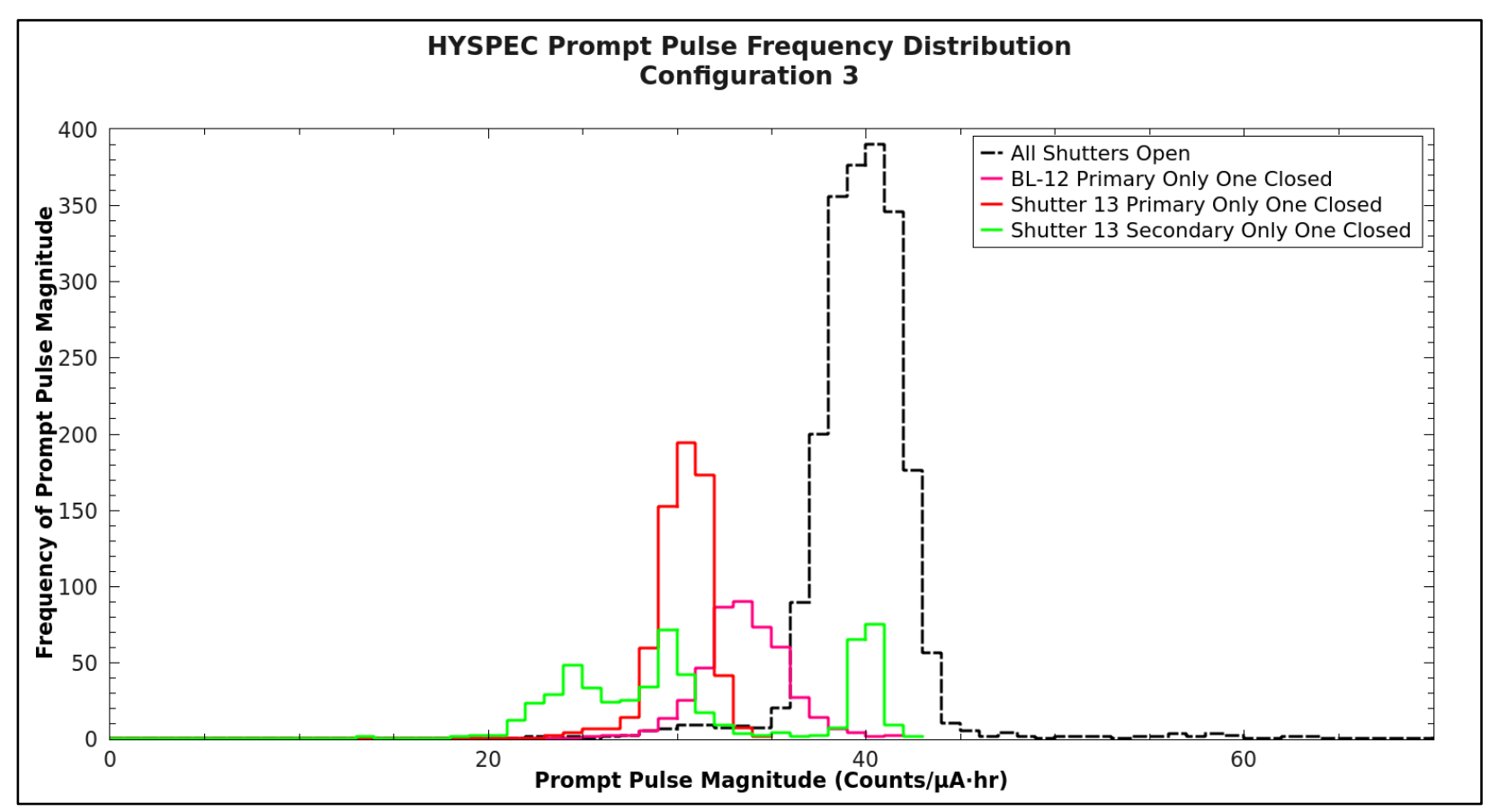

Figure 25: HYSPEC in configuration 3. Comparing background levels when all shutters on the north side of the target facility were open to when certain beamlines were the only ones closed. 


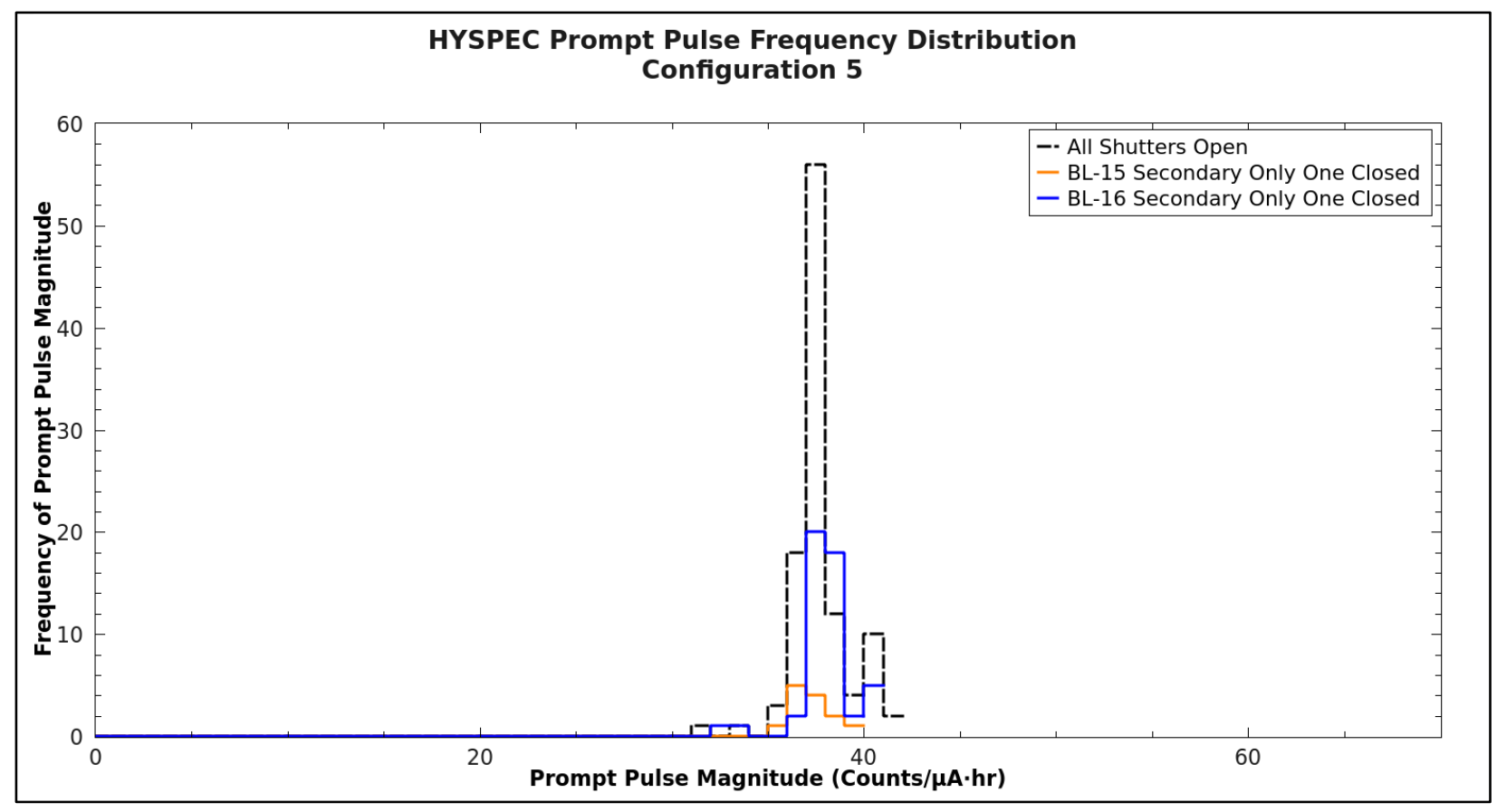

Figure 26: HYSPEC in configuration 5. Comparing background levels when all shutters on the north side of the target facility were open to when certain beamlines were the only ones closed.

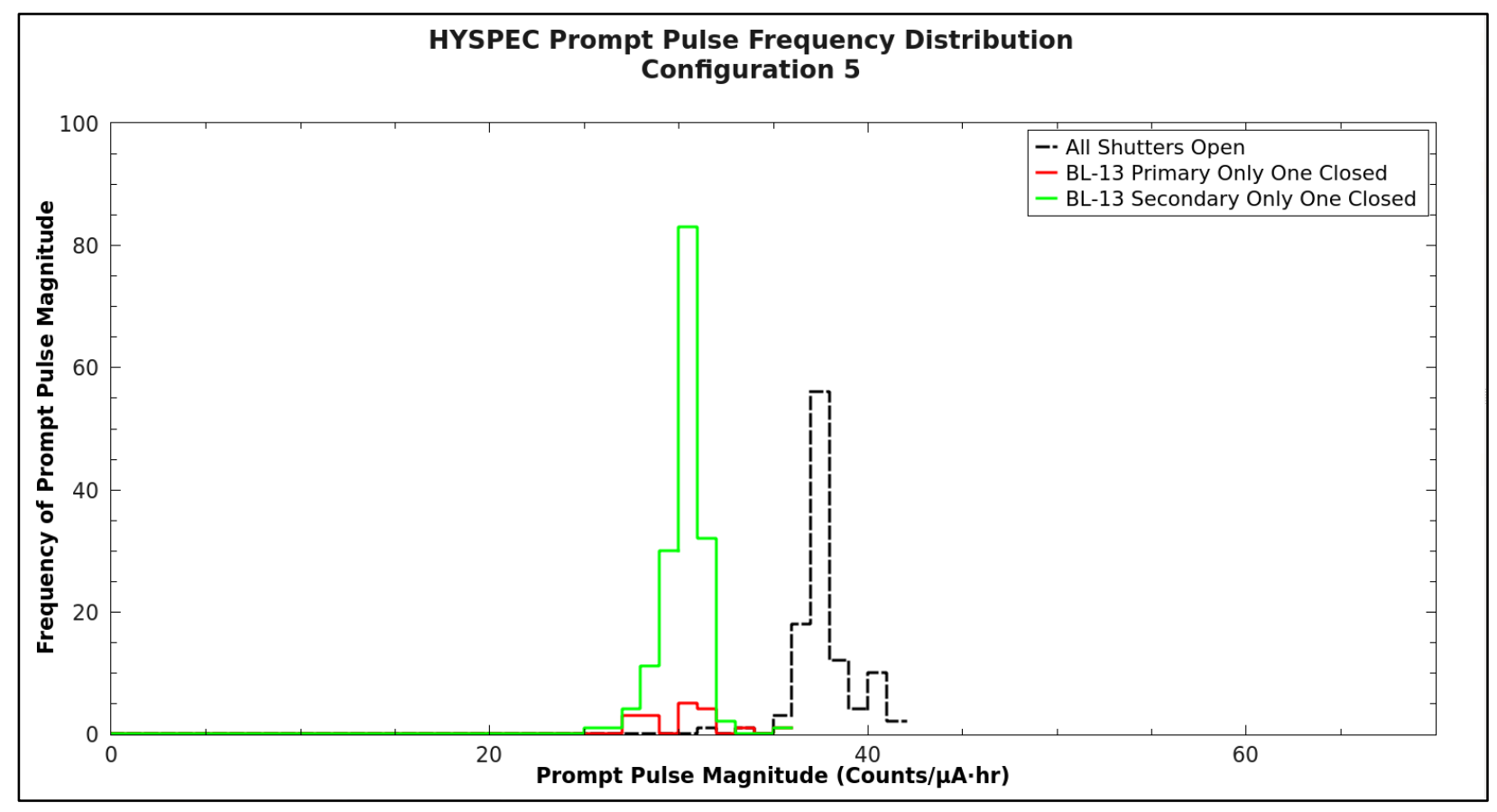

Figure 27: HYSPEC in configuration 5. Comparing background levels when all shutters on the north side of the target facility were open to when certain beamlines were the only ones closed. 


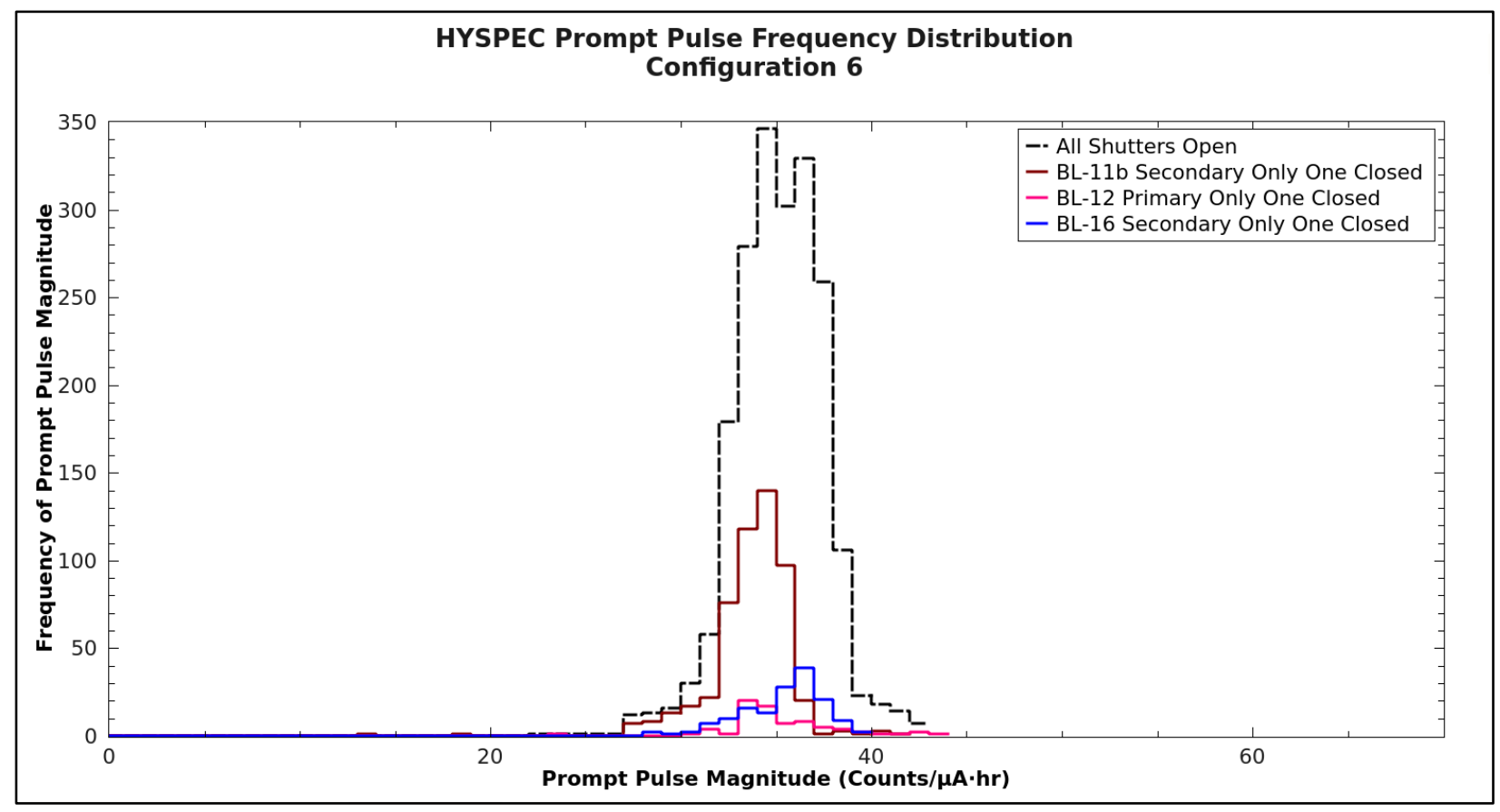

Figure 28: HYSPEC in configuration 6. Comparing background levels when all shutters on the north side of the target facility were open to when certain beamlines were the only ones closed.

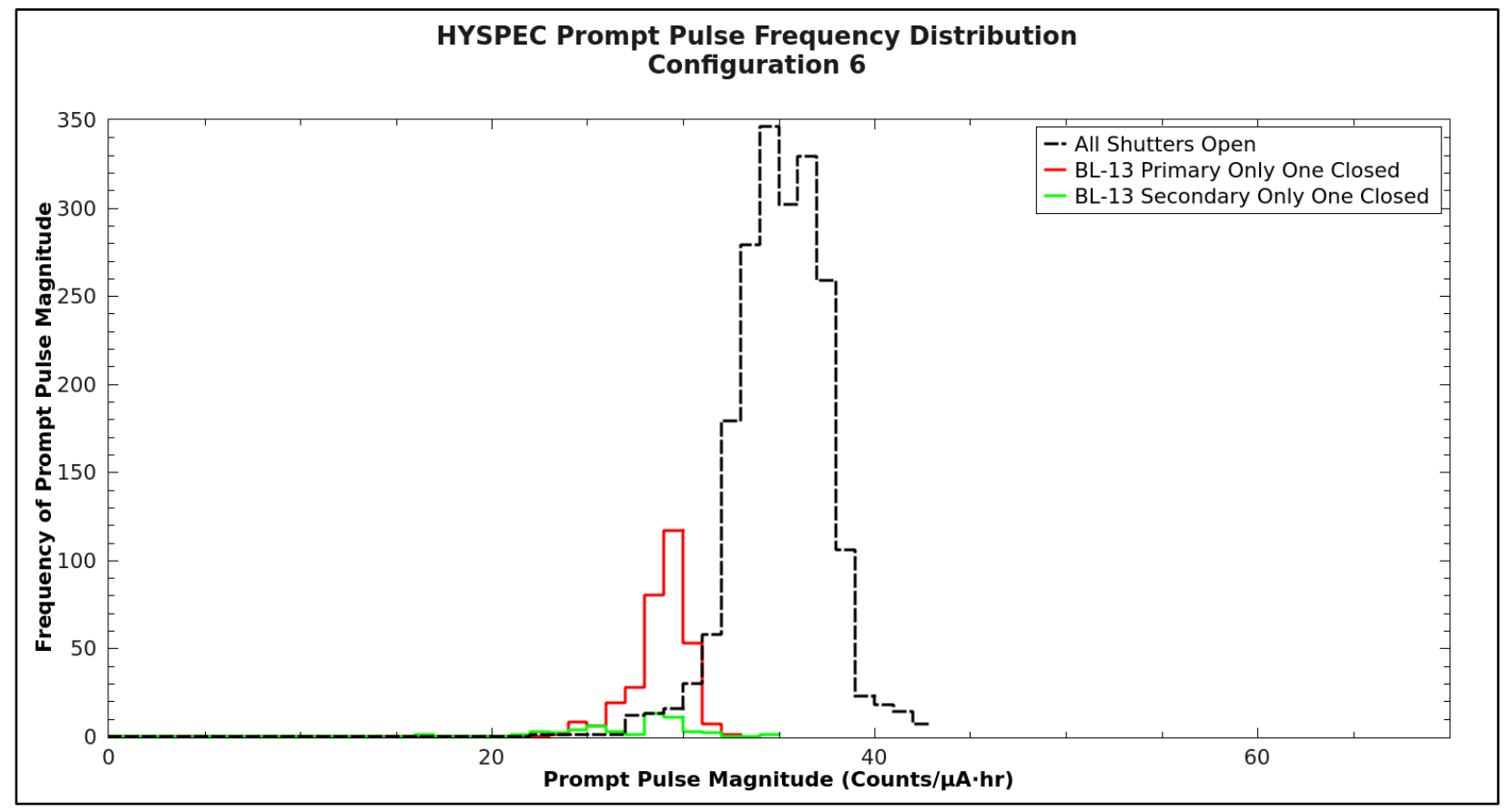

Figure 29: HYSPEC in configuration 6. Comparing background levels when all shutters on the north side of the target facility were open to when certain beamlines were the only ones closed. 


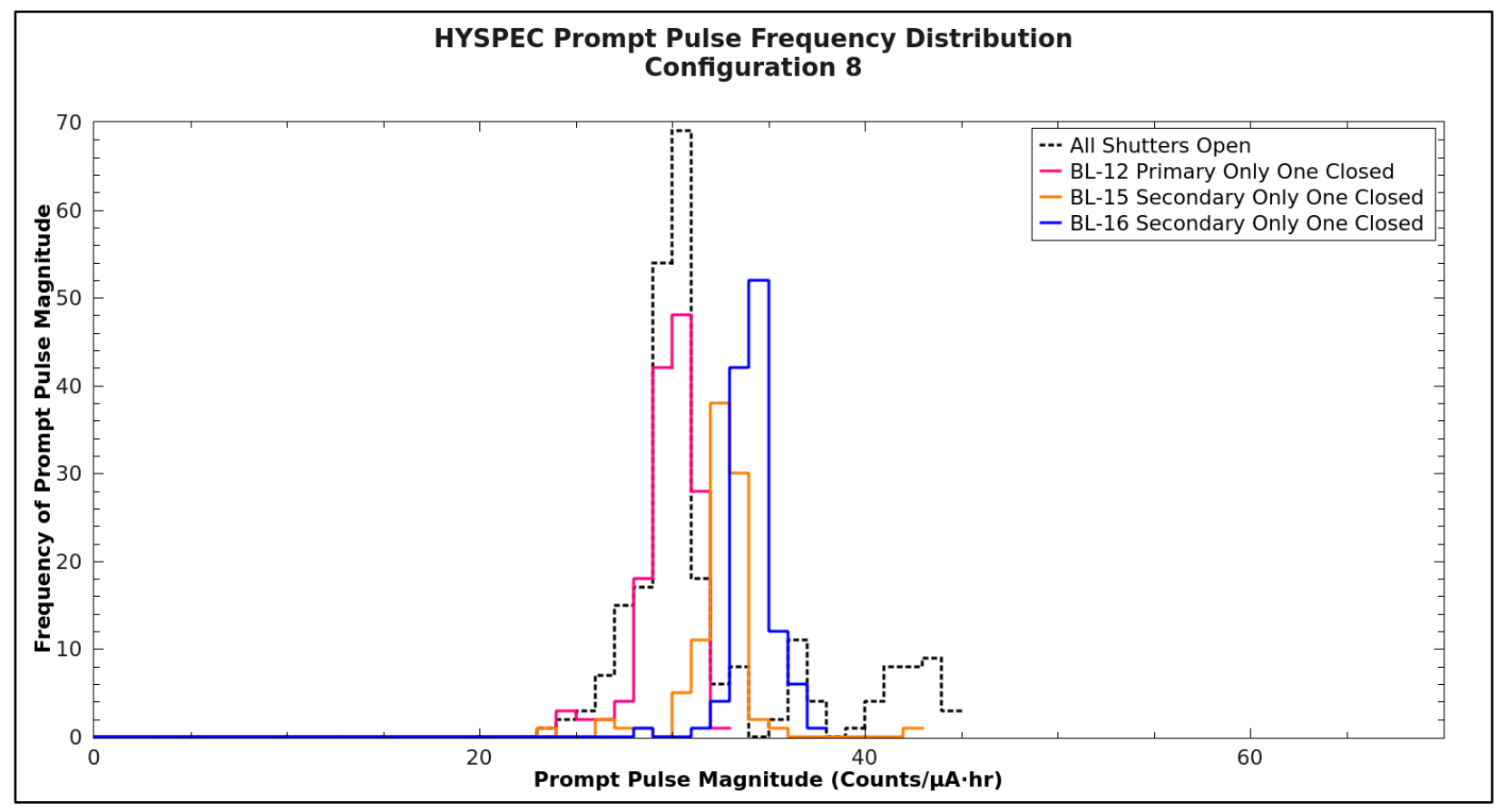

Figure 30: HYSPEC in configuration 8. Comparing background levels when all shutters on the north side of the target facility were open to when certain beamlines were the only ones closed. 



\section{APPENDIX K. PROMPT PULSE SHAPE COMPARISONS}

Representative pulse shapes were determined by taking runs only from within a specific shutter and instrument configuration. These refined run populations were then randomly sampled and averaged across each time bin in the pulse. The result of this process was an averaged pulse shape representing a very specific set of instrument and shutter configurations. This pulse shape could then be used to determine the difference between all shutters open and one shutter being the only one closed. This difference was determined by performing a simple arithmetic subtraction of one from the other on a binby-bin basis.

The statistical comparisons of the prompt pulse shapes were performed in the following manner. The prompt pulse in question was identified originally as the user data that fell within a predetermined $3,000 \mu$ s wide TOF window. This $3,000 \mu$ s wide data set was then re-binned into $50 \mu$ s bins making a total of 60 bins for each data set. To negate the effects of pulse magnitude, each pulse was normalized to

unit area. This allowed the application of the Pearson's $\mathrm{Chi}^{2}$ test to be an indicator of shape without incorporating differences in magnitude.

If the first of the two data sets to be compared is referred to as (A) and the second (B) then the Pearson's $\mathrm{Chi}^{2}$ test was applied to the two distributions in the following manner.

$$
X^{2}=\sum_{i=1}^{N} \frac{\left(A_{i}-B_{i}\right)^{2}}{\sigma_{A_{i}}^{2}+\sigma_{B_{i}}^{2}}
$$

This calculated test statistic $\left(\mathrm{X}^{2}\right)$ was then compared with standard $\mathrm{Chi}^{2}$ tables to obtain an appropriate $\mathrm{p}$-value which represents the probability that differences observed in the two distributions were caused by non-statistical influences. For example, a high p-value (in this analysis) would indicate a high probability that something other than statistical fluctuations was affecting the difference in the two distributions while a low p-value would indicate a high probability that the differences in the two distributions was largely due to statistical fluctuations alone. 

INTERNAL DISTRIBUTION

$\begin{array}{llll}\text { 1. } & \text { D.H. Abercrombie } & 9 . & \text { K.W. Herwig } \\ \text { 2. } & \text { P. Langan } & 10 . & \text { G.L. Greene } \\ \text { 3. } & \text { D.A. Tennant } & 11 . & \text { W. Lu } \\ \text { 4. } & \text { R.J. Mcqueeney } & 12 . & \text { I. Popova } \\ \text { 5. } & \text { J.D. Galambos } & 13 . & \text { ORNL Office of Technical Information } \\ \text { 6. } & \text { E.B. Iverson } & & \text { and Classification } \\ \text { 7. } & \text { F.X. Gallmeier } & 15 . & \text { All ORNL SNS Instrument Scientists } \\ \text { 8. } & \text { B. L. Winn } & & \end{array}$

\section{EXTERNAL DISTRIBUTION}

16. H. Kung, Idaho National Engineering and Environmental Laboratory, Mail Stop 7100, P.O. Box 1628, Idaho Falls, ID 83415-7101

17. J.B. Murphy, Idaho National Engineering and Environmental Laboratory, Mail Stop 7100, P.O. Box 1628, Idaho Falls, ID 83415-7101

18. J.J. Rhyne, US Department of Energy, N61, 1000 Independence Avenue SW, Forrestal Building G-050, Washington, DC 20585 